Available online at GSC Online Press Directory

GSC Biological and Pharmaceutical Sciences

e-ISSN: 2581-3250, CODEN (USA): GBPSC2

Journal homepage: https://www.gsconlinepress.com/journals/gscbps

(RESEARCH ARTICLE)

\title{
Measuring IGF-1 and IGFBP-3 profiles in women seeking assisted reproduction; relevance to clinical outcomes from in vitro fertilization (Study 5).
}

\author{
John L Yovich ${ }^{1,2,}{ }^{*}$, Syeda Zaidi ${ }^{1,3}$, Minh DK Nguyen ${ }^{1}$, and Peter M Hinchliffe ${ }^{1}$ \\ ${ }^{1}$ PIVET Medical Centre Perth, Western Australia, Australia 6007. \\ 2 Department of Pharmacy and Biomedical Sciences Curtin University Perth, Western Australia, Australia 6845. \\ ${ }^{3}$ Current address Department of Obstetrics and Gynaecology, Faculty of Medicine, University of Malaya Lembah Pantai, \\ Kuala Lumpur Malaysia 59100.
}

Publication history: Received on 03 November 2020; revised on 09 November 2020; accepted on 10 November 2020

Article DOI: https://doi.org/10.30574/gscbps.2020.13.3.0355

\begin{abstract}
In this fifth study examining the relevance of the insulin-like growth factor (IGF) profiles, namely serum IGF-1 levels, its main binding protein, serum IGFBP-3 levels and the IGF ratio (IGFBP-3/IGF-1), we have examined clinical outcomes with respect to the quartile ranges of each parameter measured in 1633 IVF-naïve women. The outcomes were derived from 1035 of these women who initiated 1747 IVF cycles. Of the three parameters, only IGF-1 levels proved relevant to the clinical outcomes. In particular, this study confirmed our earlier report that IGF-1 levels decline across the quartiles with the woman's advancing age and the proportion of women with IGF-1 levels in the highest quartile comprised the younger, whilst those women $\geq 40$ years were mostly in the lowest quartile $(p<0.0001)$. IVF cycle cancellations were not different across the quartiles but at oocyte pick-up (OPU) there were significantly more oocytes from the highest IGF-1 quartile (10.4 oocytes) compared to the lowest ( 8.9 oocytes; $\mathrm{p}<0.01)$. Furthermore, the proportion of oocytes shown to be at the mature MII stage were also significantly higher $(p=0.01)$. Consequently, the oocyte utilization rate was significantly greater from those women with the highest IGF-1 levels generating 5.3 embryos vs 4.3 embryos for the lowest quartile. However, the utilization rate of those embryos generated were similar across the IGF-1 quartiles and there were similar rates of "freeze-all embryos", albeit more women had increased monitoring for ovarian hyperstimulation risk, related to the higher egg numbers in the highest IGF-1 quartile. The most striking clinical outcomes were that of the pregnancy productivity rate (including fresh and frozen embryo transfers from a single OPU) which was greatest in the highest IGF-1 quartile (61.5\% vs $48.2 \%$ per OPU across all ages p<0.0002). Miscarriage rates were not different but live birth productivity rates were significantly greatest for the highest IGF-1 quartile (49\% vs $33 \%$ per OPU across all ages; $\mathrm{p}<0.0002$ ). We conclude that a preliminary measurement of the IGF-1 level can help to define the poor-prognosis female prior to IVF treatment and on whom adjuvant studies can be focused.
\end{abstract}

Keywords: Growth Hormone (GH); Insulin-Like Growth Factor (IGF). IGF-1 Profile; IGFBP-3/ IGF-1 Ratio (IGF Ratio); Assisted Reproductive Technology (ART); In Vitro Fertilization (IVF); Growth Hormone (GH); Poor Prognosis Female; Pregnancy Productivity Rate; Live Birth Productivity Rate.

\section{Introduction}

Although assisted reproductive technologies (ART) have generated an estimated 10 million children from sub-fertile settings, the successful cases have occurred mainly in younger women, particularly those aged $<35$ years. Currently, most women presenting for ART are aged between 35-40 years and, although the prognosis is markedly reduced, an increasing proportion of women $>40$ years are presenting, currently $24 \%$ of cases in the year 2018 in Australia and New

\footnotetext{
${ }^{*}$ Corresponding author: John L Yovich

PIVET Medical Centre Perth, Western Australia Australia 6007.
}

Copyright (C) 2020 Author(s) retain the copyright of this article. This article is published under the terms of the Creative Commons Attribution Liscense 4.0. 
Zealand [1]. However, there are many successful outcomes in this older group utilizing both autologous as well as donor oocytes, however, defining the poor-prognosis woman is reliant upon undergoing one or more attempts to determine responsiveness to ovarian stimulation. This is partially predicted by preliminary measurements of ovarian reserve, namely by antral follicle counts (AFC) and/or by anti-Mullerian hormone (AMH) estimations [2]. However, it is our longstanding experience that these measures simply determine the woman's responsiveness to stimulation by gonadotrophin hormones, namely follicle stimulating hormone (FSH) and luteinizing hormone (LH), and this is mostly overcome by using higher dosages and repeated treatment cycles [3]. Applying PIVET FSH-dosing algorithms [4,5], women may respond across the range of 50 IU daily up to a maximum of 450 IU daily with successful live births across the range. Whilst most poor-prognosis scenarios occur among women $>40$-years, many exhibit this problem at younger ages, and not always with determinations of low ovarian reserve. Such patients may be offered one or several of more than 50 different adjuvants [6,], none of which have high-level evidence supporting their use [7,8]. One such adjuvant with some favorable supporting studies is that of growth hormone, but so far this has been trialed on an empirical basis, without any definitive appraisal to define its potential benefit $[6,9,10,11]$. Nonetheless, the notion of adult growth hormone deficiency (AGHD) is appealing [9], however, making such a diagnosis is a complex exercise unless a screening test can define which woman should be investigated for such a potential diagnosis. In children, the application of the IGF profile is undertaken on those whose stature is below the $5^{\text {th }}$ centile along with reduced growth velocity and delayed bone age on radiographic assessment [12,]. The main test is insulin-like growth factor (IGF-1) and this is usually tested along with its main binding protein IGFBP-3 (one of six) enabling the calculation of an IGF ratio, intended to provide a better index of bioavailability of IGF-1 [13]. The binding protein acts to prolong IGF-1 activity significantly [14,15]. In adults, differentiating normal aging effects from AGHD is only recently being entertained by Endocrine societies around the world, and requires appropriate provocative challenge testing for clarification [16,17]. In order to investigate the idea of the IGF profile providing a prognosis for IVF-naïve women, we have undertaken a sequence of studies to explore its relevance $[18,19,20,21]$. This fifth study closely examines the IGF profile to clinical outcomes among women who have been carefully screened prior to undertaking IVF-related procedures, then documenting outcomes according to their underlying IGF profile.

\section{Material and methods}

This is the fifth study derived from a data set at the PIVET program for ART in Perth, Australia which was established in 1981 [22,23]. From 2001, all ART treatment cycles, numbering 23,509, have been comprehensively recorded in a validated data base using Filemaker Pro (Apple Inc, USA). Since January 2011, the ART program has been characterized by three important developments, firstly, encouraging all ART-naïve women to undertake a preliminary Assessment Cycle (AC) [5]; secondly, utilizing the well described PIVET dosing-algorithms for ovarian stimulation [24], one of which is shown in Table 1; and thirdly, a commitment to single embryo transfer (SET) procedures for both fresh and frozen embryos across the entire age profile. Currently, in keeping with a widely encouraged practice across Australia and New Zealand, SET procedures at PIVET now exceed 95\% of all cases [1]. In association with this, PIVET commits to a blastocyst culture system whenever $\geq 3$ embryos are progressing on a Day-3 laboratory inspection, currently occurring in $90 \%$ of in vitro fertilization (IVF) cases. This means that there is also a high commitment to cryopreservation, which at PIVET is conducted using the Cryotop (Kitazato, Tokyo, Japan) vitrification technique [25].

Across the period January 2011 to December 2019, 3751 women entered into 10,728 treatments of various ART categories. Figure 1 shows the derivation of 1633 women from a total 2319 women who had an AC which also included an insulin-like growth factor (IGF) profile comprising IGF-1 along with its main binding protein IGFBP-3, and a calculation of IGF ratio being the division of IGF-1 into the higher concentration IGFBP-3 level. Our earlier studies examined the IGF profile against clinical parameters including age, body mass index (BMI) and body stature (Study 1) [18] as well as estimations of ovarian reserve by an antral follicle count (AFC) along with serum AMH testing (Study 2) [19]. All these tests were performed around Day $-5 \pm 1$ of the menstrual cycle, in the morning following a minimal "tea and toast" breakfast. These ACs were one-off evaluations of the underlying infertility factors and not repeated between ART treatments. 
Table 1 One of the PIVET FSH-dosing Algorithms which have been applied throughout this study and which covers for $12.5 \mathrm{IU}$ and $25 \mathrm{IU}$ increments of FSH which is most suited to the Gonal-F (Gonal-F (follitropin alfa; Merk Healthcare, New South Wales, Australia) pen and biosimilars. It also suits the Puregon (follitropin beta; Merck Sharp \& Dohme, New South Wales, Australia) pen at higher dosages $\geq 300$ IU. The long-acting Elonva (corifollitropin alfa; Merck Sharp \& Dohme, New south Wales, Australia) can be safely used in those women denoted by FSH dosages in the green sector.

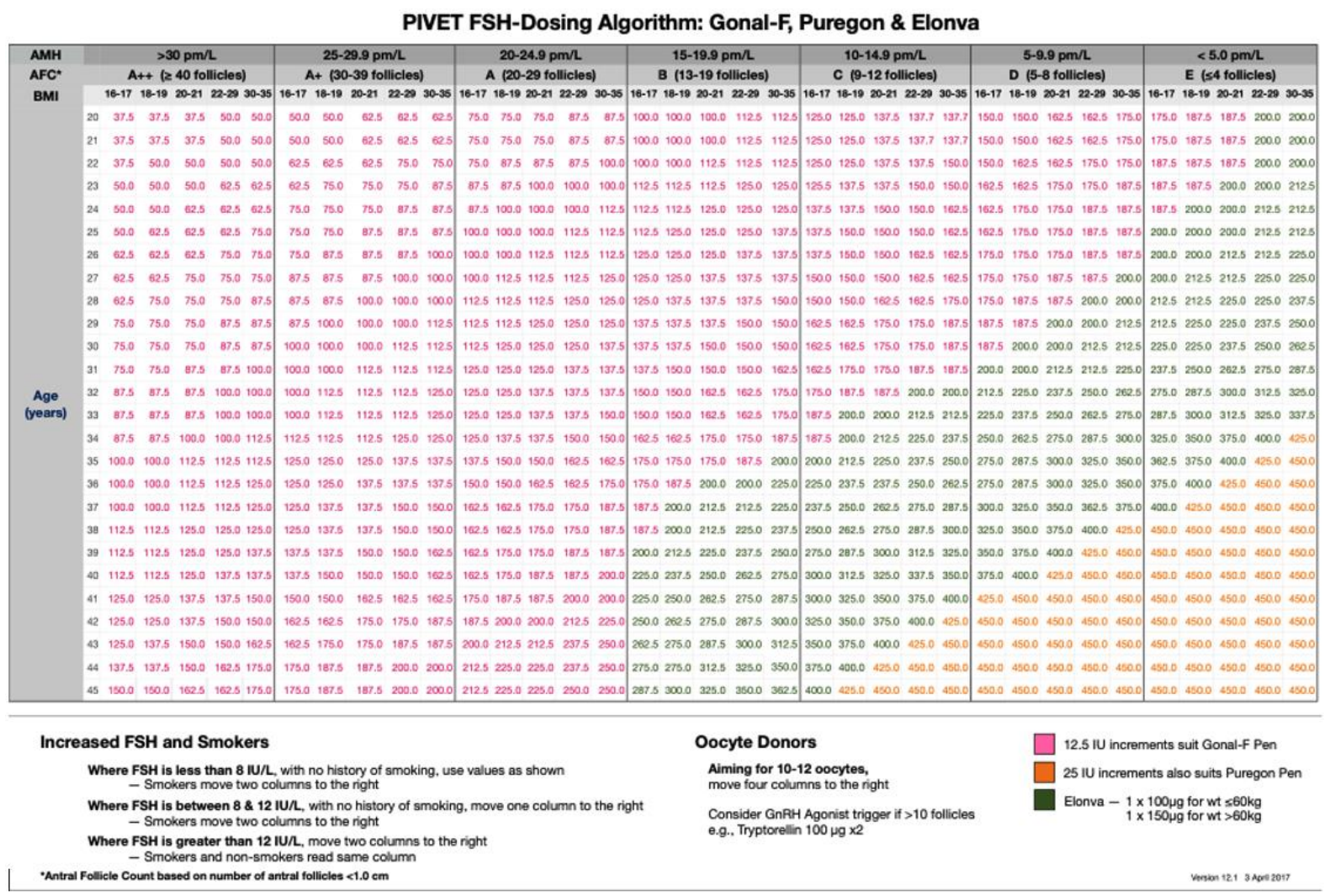

In the current study we are exploring the relevance of the IGF profile to subsequent IVF treatment outcomes. From Figure 1 it can be seen that 1035 of the women who completed an AC with full IGF profile proceeded to a total 1747 IVF treatments, utilizing intra-cytoplasmic sperm injection (ICSI) according to well reported PIVET protocols where indicated [26]. This included an IVF-ICSI Split model for unexplained infertility cases. Those IVF treatment cycle outcomes are categorized according to the IGF quartiles which were established in Study 1, with the ranges (lowest quartile, inter-quartile; IQR and highest quartile) for IGF-1, IGFBP-3 and the IGF ratio detailed in Table 2 which is reproduced from Study 1.

Our studies have applied SI units (Système Internationale; International System) for the IGF profile (reporting nmol/L) rather than conventional units $(\mathrm{ng} / \mathrm{mL}$ ) which requires a conversion factor of 7.65 ; hence $25 \mathrm{nmol} / \mathrm{L}$ IGF-1 can be read as $191 \mathrm{ng} / \mathrm{mL}$ IGF-1. Furthermore, it has been our long-sanding convention to report the IGF ratio as IGFBP-3 (a higher concentration) divided by IGF-1 (a lower concentration), meaning the ratio is in larger numbers such as $160 / 25 ; 6.4$ rather than fractions such as 25/160; 0.16. For those desiring the fraction expression this means inverting the numerator/ denominator. Our convention means the interquartile range for IGF ratio is 5.7 to 7.8 (same as 0.18 to 0.13 ). From Table 2 the range of IGF profile measures; 2.5 to 20.4 can be converted to 0.4 to 0.05 . PIVET convention means that higher IGF-1 levels associate with lower IGF ratios; whereas the alternative displays both IGF-1 and IGF ratio measuring in the same direction e.g. high levels with high ratios and low levels with low ratios. 


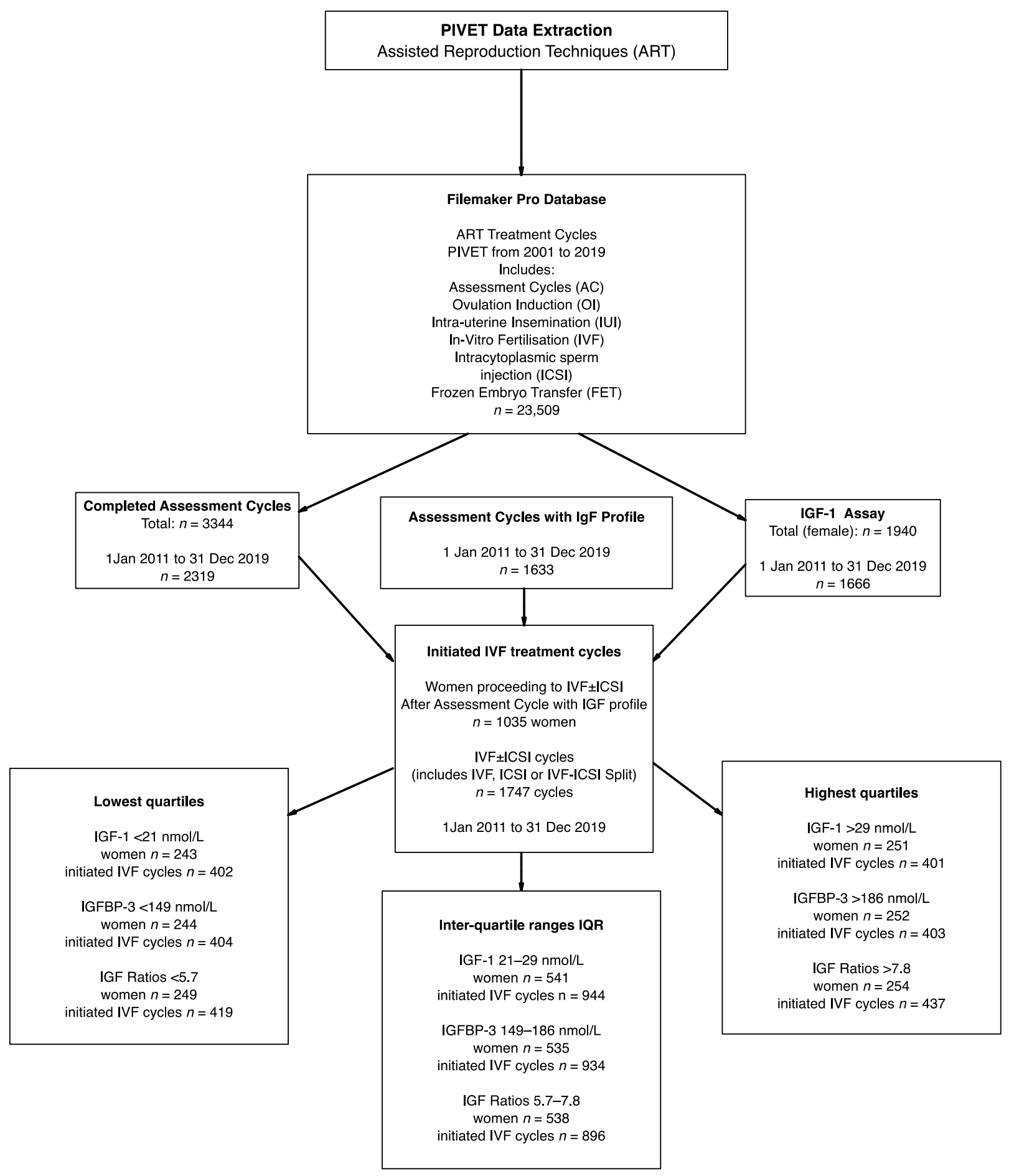

Figure 1 Flow diagram showing derivation of 1035 women IVF-naïve women who had completed an Assessment Cycle which included an IGF profile and who subsequently initiated 1747 IVF \pm ICSI treatment cycles. 
Table 2 Summary statistics of 1633 infertile, but IVF-naïve, women who completed an IGF profile (embracing IGF-1, IGBP-3 and the ratio IGBP-3/IGF-1) during an Assessment Cycle. Reproduced from Study 1 [18].

\begin{tabular}{|l|l|l|l|}
\hline \multicolumn{2}{|l|}{ Complete IGF profile - summary statistics } \\
\hline & IGF-1 nmol/L & IGFBP-3 nmol/L & IGFBP-3/ IGF-1 Ratio \\
\hline N & 1633 & 1633 & 1633 \\
\hline Min & 8 & 59 & 2.5 \\
\hline Max & 63 & 270 & 20.4 \\
\hline Sum & 41707 & 273729 & 11287.5 \\
\hline Mean & 25.54 & 167.6 & 6.91 \\
\hline Std. error & 0.17 & 0.73 & 0.04 \\
\hline Variance & 47.67 & 865.72 & 3.20 \\
\hline Stand. dev & 6.9 & 29.42 & 1.79 \\
\hline Median & 25 & 166 & 6.6 \\
\hline $25^{\text {th }}$ percentile & 21 & 148.5 & 5.7 \\
\hline $75^{\text {th }}$ percentile & 29 & 186 & 7.8 \\
\hline Skewness & 0.58 & 0.23 & 1.41 \\
\hline Kurtosis & 390.92 & 334.53 & 747.58 \\
\hline Geom. mean & 24.61 & 164.99 & 6.71 \\
\hline Coeff. var & 27.03 & 17.55 & 25.89 \\
\hline
\end{tabular}

\subsection{IVF outcome parameters}

\subsubsection{Cycles initiated}

denotes those autologous IVF cycles which commence ovarian stimulation with gonadotrophins on Day-3. Approval requires that base-line parameters are met on day-2, namely serum gonadotrophins, namely follicle stimulating hormone (FSH) and luteinizing hormone (LH) indicating the woman is not menopausal or in ovarian failure. In addition, her ovarian hormones must be at basal levels, namely estradiol (E2) level $<150 \mathrm{pmol} / \mathrm{L}$ and Progesterone (P4) level $<5$ nmol/L along with her pituitary Prolactin <750 IU/L [5].

\subsubsection{Cancelled cycle}

indicates the IVF cycle is abandoned prior to HCG Trigger, usually given around day-12. This usually arises from a lack of response to the ovarian stimulation with lack of E2 elevation and failure to detect ovarian follicles $\geq 16 \mathrm{~mm}$ by transvaginal pelvic ultrasound. Cycles with poor responses are maximally stimulated with follicle stimulating hormone (FSH) at $450 \mathrm{IU} /$ day up to a maximum 16 days (day-19 of IVF cycle). Cycles may also be abandoned if there is evidence of premature ovulation (Rising LH, elevated P4 and marked fall in E2). This problem is unusual in current practice which routinely applies gonadotrophin releasing hormone (GNRH) antagonists [5]. Occasional causes of cancelled cycles may be patient-related issues such as intercurrent illness, domestic stresses and logistical problems related to travel from remote locations.

\subsubsection{Oocytes retrieved}

denotes every oocyte detected following ovarian follicle aspiration at ovum pick-up (OPU). At PIVET this is undertaken by a single-lumen aspirating needle when follicle numbers $>12 \mathrm{~mm} \geq 5$; and a double-lumen flushing needle when there are fewer follicles. 


\subsubsection{Oocyte utilization rate}

denotes the number of oocytes which fertilize and contribute to the formation of embryos which are utilized in a fresh embryo transfer (ET) procedure or a frozen embryo transfer (FET) procedure after a period of cryopreservation. This is factored for all oocytes, be they at MII, MI or the germinal vesicle stage, or even if they are shown to have degenerated or have fractured zona pellucidae.

\subsubsection{Freeze-all embryos}

denotes those cases where a fresh embryo transfer is not performed, mainly to reduce the risk of ovarian hyperstimulation syndrome (OHSS) and suitable embryos, mostly blastocysts are committed to cryopreservation for future FETs. This option is considered when $\geq 15$ oocytes are recovered at OPU or fresh ETs are deferred for other reasons (including when the woman is unwell, or embryo transfer is deferred awaiting procedures such as hysteroscopy or laparoscopy to correct pelvic conditions).

\subsubsection{Increased monitoring}

relates to those women who have $>12$ oocytes recovered at OPU. Such women have daily contact with PIVET to discuss the woman's wellbeing, including measurement of her abdominal girth, description of urine characteristics (light or dark color and output), as well as blood test monitoring every $2^{\text {nd }}$ or $3^{\text {rd }}$ day of the luteal phase, with concern if E2 $>6000 \mathrm{pm} / \mathrm{L}$ and/or P4 $>600 \mathrm{~nm} / \mathrm{L}$. Such patients may return to PIVET for IV fluids and specific gravity monitoring of their urine. Occasional women will need paracentesis of ascites and hospitalization, although such events have reduced to $0.1 \%$ in recent years.

\subsubsection{Embryo utilization rate}

denotes the number of fertilized oocytes (defined at the two-pronuclear; 2PN stage), which are then utilized in a fresh ET procedure or subsequently in a FET procedure after a period of cryopreservation. PIVET protocols [26] means that $\sim 85 \%$ of all oocytes retrieved undergo a corona-cumulus stripping process for ICSI, which enables identification of the maturational stage of the oocyte, particularly the MII oocyte which has released a single polar body. Those oocytes subjected to IVF-only, have the maturational stage presumed at the 18-hour PN-stage check when pipette-stripping occurs. If the 2-PN stage is identified, it is presumed that the oocyte must have been at the MII stage at OPU.

\subsubsection{Pregnancy productivity rate}

denotes the total number of pregnancies arising after both fresh ETs and FETs related to a single initiated cycle reaching the stage of OPU. This means the freeze-all or freeze-best embryo strategies do not prejudice the "pregnancy rate". It is for this reason we have developed such terminology in rather than the oft-used cumulative pregnancy rate which, traditionally related to a number of IVF/OPU cycles [27].

\subsubsection{Miscarriage rate}

denotes those pregnancies which do not advance to a livebirth and are invariably lost before 20-weeks; the division point for obstetric outcomes. At PIVET pregnancies are diagnosed provisionally at Day-19 of the luteal phase as "4weeks" when serum BhCG is detected $>25 \mathrm{IU} / \mathrm{l}$. At this stage the pregnancy is "biochemical only" but is tracked each week until a transvaginal pelvic scan at 7-weeks denotes the presence of an intra-uterine gestational sac, expectantly with a definable viable fetus. However, at PIVET clinical pregnancy is diagnosed at week- 5 if the BHCG elevation is around 5 to 10 -fold and at week- 6 if there is a further 2 to 5 -fold rise along with associated appropriate levels of E2 and P4. PIVET has hormonal support strategies for those pregnancies with threatened miscarriage and suboptimal P4 levels [5]. Miscarriage rates include pregnancies of unknown location, ectopic gestations, blighted ovum losses and terminations of abnormal or demised fetuses prior to 20 weeks. In this study the miscarriage rate is given as all pregnancy losses (numerator) as a proportion of clinical pregnancies (denominator).

\subsubsection{Livebirth productivity rate}

denotes the total number of pregnancies arising after both fresh ETs and FETs related to a single initiated cycle reaching the stage of OPU and delivering after 20 weeks. As with pregnancy rates, this means freeze-all or freeze-best embryo strategies do not prejudice the "pregnancy rate". It is for this reason we have developed such terminology in rather than the oft-used cumulative live birth rate which, traditionally related to a number of IVF/OPU cycles [27]. Each delivery is counted as one livebirth, even where twins or higher-order multiples are delivered. Stillbirths are not included as a live birth, but perinatal losses are included. Fortunately, such adverse outcomes are now uncommon in Australia due to the SET policy [1] and PIVET records twinning rates at 2.7\%, nil triplets or higher order multiples in recent years. 


\subsection{Statistics}

Data extractions from the Filemaker Pro (Apple Inc, USA) database were placed in Microsoft Excel spreadsheets and sorted according to the relevant tests. Thereafter the sorted data was placed in the application Past 4.03 (developed by Øyvind Hammer) [28] for statistical data analysis. This application also generated the Tables comprising the statistical summaries, finally placed in Microsoft Word for clearer display. Having demonstrated that the data comprising the IGF profile (IGF-1, IGFBP-3 and IGF Ratio) are all distributed in a Normal fashion, the relationship among the means was examined by one-way ANOVA for overall comparison. The Kruskal-Wallis test was applied to examine equality between sample medians and Mann-Whitney applied for pairwise comparisons between individual sub-groups. Ratio comparisons between two groups were analyzed in 2x2 contingency tables, mainly by Fisher's exact test, or by Chisquared applying Yates' continuity correction factor for the larger data sets. Following corrections, probability values of $\mathrm{p}<0.01$ were considered significant for any test. As this data is retrospective by design, with wide variance and large kurtosis, hence several comparisons which were borderline, ranging 0.03 to 0.06 ; were classified as being of uncertain significance hence not claimed as significant. The Figures displayed for this study are derived from Excel v 16.42 (2020).

\section{Results}

A summary of key outcome measures with respect to IGF-1 is shown in Table 3 (3a; depicting raw data and Table 3b; depicting rates), providing the data from which the Figures 2-7 are derived. A summary of key outcome measures with respect to IGFBP-3 is shown in Table 4 (4a; depicting raw data and Table $4 \mathrm{~b}$; depicting rates). A summary of key outcome measures with respect to the IGF ratio (IGFBP-3/IGF-1) is shown in Table 5 (5a; depicting raw data and Table 5 b; depicting rates).

Table 3a Tabulates the raw data for the IGF-1 quartiles for the key outcome measures of the 1035 women who initiated 1747 IVF treatment cycles.

\begin{tabular}{|c|c|c|c|c|c|c|c|c|c|c|c|c|c|c|c|}
\hline \multirow{2}{*}{$\begin{array}{l}\text { IGF-1 levels } \\
\text { Age Groups years }\end{array}$} & \multicolumn{5}{|c|}{$<21 \mathrm{nmol} / \mathrm{L}$} & \multicolumn{5}{|c|}{ 21-29 nmol/L } & \multicolumn{5}{|c|}{$>29 \mathrm{nmol} / \mathrm{L}$} \\
\hline & $<35$ & 35-39 & $40-44$ & $\geq 45$ & Total & $<35$ & $35-39$ & $40-44$ & $\geq 45$ & Total & $<35$ & 35-39 & $40-44$ & $\geq 45$ & Total \\
\hline Initiated Cycles & 120 & 144 & 114 & 25 & 403 & 411 & 299 & 180 & 60 & 950 & 229 & 119 & 51 & 3 & 402 \\
\hline Patients Represented & 83 & 91 & 53 & 17 & 244 & 261 & 166 & 90 & 27 & 544 & 161 & 62 & 25 & 3 & 251 \\
\hline Cancelled cycles & 14 & 13 & 17 & 2 & 46 & 36 & 30 & 19 & 14 & 99 & 21 & 8 & 7 & 2 & 38 \\
\hline \#OPU & 106 & 131 & 97 & 23 & 357 & 375 & 269 & 161 & 46 & 851 & 208 & 111 & 44 & 1 & 364 \\
\hline Oocytes Collected & 1214 & 1235 & 662 & 82 & 3193 & 4297 & 2531 & 1224 & 220 & 8272 & 2473 & 1084 & 276 & 5 & 3838 \\
\hline Minimum Oocytes/OPU & 1 & 1 & 1 & 1 & 1 & 1 & 1 & 1 & 1 & 1 & 1 & 1 & 1 & 5 & 1 \\
\hline Maximum Oocytes/OPU & 36 & 26 & 23 & 13 & 36 & 36 & 39 & 30 & 16 & 39 & 36 & 25 & 18 & 5 & 36 \\
\hline Std Deviation (oocytes) & 7.3 & 5 & 5 & 3 & 20.3 & 6.5 & 5.7 & 5.2 & 4 & 21.4 & 5.9 & 6 & 5 & 0 & 16.2 \\
\hline Increased Monitoring & 42 & 33 & 13 & 1 & 89 & 137 & 65 & 28 & 5 & 235 & 76 & 34 & 6 & 0 & 116 \\
\hline Oocytes Injected & 761 & 787 & 455 & 68 & 2071 & 2760 & 1664 & 826 & 144 & 5394 & 1550 & 696 & 180 & 5 & 2431 \\
\hline$\# 2 \mathrm{PN}$ & 672 & 690 & 331 & 53 & 1746 & 2480 & 1420 & 644 & 105 & 4649 & 1438 & 572 & 144 & 5 & 2159 \\
\hline \#2PN (ICSI Only) & 594 & 563 & 303 & 47 & 1507 & 2079 & 1237 & 566 & 101 & 3983 & 1232 & 503 & 124 & 5 & 1864 \\
\hline \#Freeze-all embryos & 15 & 11 & 13 & 5 & 44 & 46 & 45 & 17 & 7 & 115 & 26 & 12 & 4 & 0 & 42 \\
\hline \#ET cycles & 79 & 112 & 65 & 14 & 270 & 314 & 193 & 131 & 25 & 663 & 176 & 86 & 32 & 1 & 295 \\
\hline \#Fresh embryos transf. & 80 & 118 & 84 & 22 & 304 & 328 & 216 & 180 & 34 & 758 & 182 & 95 & 45 & 2 & 324 \\
\hline \#Embryos Frozen (f) & 264 & 216 & 60 & 7 & 547 & 905 & 480 & 125 & 17 & 1527 & 514 & 197 & 22 & 0 & 733 \\
\hline \#FET Cycles (f) & 111 & 81 & 32 & 6 & 230 & 291 & 197 & 74 & 11 & 573 & 181 & 84 & 11 & 0 & 276 \\
\hline \#FET embryos transf. & 111 & 85 & 34 & 7 & 237 & 298 & 209 & 77 & 14 & 598 & 183 & 82 & 12 & 0 & 277 \\
\hline Total ETs F/f & 191 & 203 & 118 & 29 & 541 & 626 & 425 & 257 & 48 & 1356 & 365 & 177 & 57 & 2 & 601 \\
\hline Total Embryos Utilized & 344 & 334 & 144 & 29 & 851 & 1233 & 696 & 305 & 51 & 2285 & 696 & 292 & 67 & 2 & 1057 \\
\hline \#Pregs IVF only & 35 & 38 & 12 & 1 & 86 & 127 & 54 & 19 & 3 & 203 & 72 & 23 & 3 & 0 & 98 \\
\hline \#Pregnancies FET & 46 & 31 & 9 & 0 & 86 & 142 & 79 & 17 & 1 & 239 & 86 & 36 & 4 & 0 & 126 \\
\hline \#Pregs F/f & 81 & 69 & 21 & 1 & 172 & 269 & 133 & 36 & 4 & 442 & 158 & 59 & 7 & 0 & 224 \\
\hline \#Miscarriages & 4 & 9 & 6 & 0 & 19 & 33 & 14 & 11 & 2 & 60 & 12 & 13 & 2 & 0 & 27 \\
\hline \#LiveBirths Fresh & 33 & 30 & 5 & 1 & 69 & 98 & 37 & 10 & 2 & 147 & 66 & 15 & 0 & 0 & 81 \\
\hline \#Livebirths FET & 32 & 25 & 7 & 0 & 64 & 108 & 56 & 10 & 1 & 175 & 65 & 30 & 3 & 0 & 98 \\
\hline \#LiveBirths F/f & 65 & 55 & 12 & 1 & 133 & 206 & 93 & 20 & 3 & 322 & 131 & 45 & 3 & 0 & 179 \\
\hline
\end{tabular}


Table 3b Tabulates the data for the IGF-1 quartiles as rates for the key outcome measures of the 1035 women who initiated 1747 IVF treatment cycles.

\begin{tabular}{|c|c|c|c|c|c|c|c|c|c|c|c|c|c|c|c|}
\hline IGF-1 levels & & & $1 \mathrm{nmol}$ & & & & & $29 \mathrm{nmo}$ & & & & & $29 \mathrm{nmol}$ & & \\
\hline Age Groups years & $<35$ & 35-39 & $40-44$ & $\geq 45$ & Total & $<35$ & 35-39 & $40-44$ & $\geq 45$ & Total & $<35$ & 35-39 & $40-44$ & $\geq 45$ & Total \\
\hline Cancellation Rate & $11.7 \%$ & $9.0 \%$ & $14.9 \%$ & $8.0 \%$ & $11.4 \%$ & $8.8 \%$ & $10.0 \%$ & $10.6 \%$ & $23.3 \%$ & $10.4 \%$ & $9.2 \%$ & $6.7 \%$ & $13.7 \%$ & $66.7 \%$ & $9.5 \%$ \\
\hline Oocytes/OPU & 11.5 & 9.4 & 6.8 & 3.6 & 8.9 & 11.5 & 9.4 & 7.6 & 4.8 & 9.7 & 11.9 & 9.8 & 6.3 & 5.0 & 10.5 \\
\hline Fert Rate (IVF \pm ICSI) & $55.4 \%$ & $55.9 \%$ & $50.0 \%$ & $64.6 \%$ & $54.7 \%$ & $57.7 \%$ & $56.1 \%$ & $52.6 \%$ & $47.7 \%$ & $56.2 \%$ & $58.1 \%$ & $52.8 \%$ & $52.2 \%$ & $100 \%$ & $56.3 \%$ \\
\hline Fert Rate (ICSI) & $48.9 \%$ & $45.6 \%$ & $45.8 \%$ & $57.3 \%$ & $47.2 \%$ & $48.4 \%$ & $48.9 \%$ & $46.2 \%$ & $45.9 \%$ & $48.2 \%$ & $49.8 \%$ & $46.4 \%$ & $44.9 \%$ & $100 \%$ & $48.6 \%$ \\
\hline Preg Rate (Fresh) & $29.2 \%$ & $26.4 \%$ & $10.5 \%$ & $4.0 \%$ & $21.3 \%$ & $30.9 \%$ & $18.1 \%$ & $10.6 \%$ & $5.0 \%$ & $21.4 \%$ & $31.4 \%$ & $19.3 \%$ & $5.9 \%$ & $0.0 \%$ & $24.4 \%$ \\
\hline Preg Rate (Frozen) & $41.4 \%$ & $38.3 \%$ & $28.1 \%$ & $0.0 \%$ & $37.4 \%$ & $48.8 \%$ & $40.1 \%$ & $23.0 \%$ & $9.1 \%$ & $41.7 \%$ & $47.5 \%$ & $42.9 \%$ & $36.4 \%$ & $0.0 \%$ & $45.7 \%$ \\
\hline Livebirth Rate (Fresh) & $27.5 \%$ & $20.8 \%$ & $4.4 \%$ & $4.0 \%$ & $17.1 \%$ & $23.8 \%$ & $12.4 \%$ & $5.6 \%$ & $3.3 \%$ & $15.5 \%$ & $28.8 \%$ & $12.6 \%$ & $0.0 \%$ & $0.0 \%$ & $20.1 \%$ \\
\hline Livebirth Rate (Frozen) & $28.8 \%$ & $30.9 \%$ & $21.9 \%$ & $0.0 \%$ & $27.8 \%$ & $37.1 \%$ & $28.4 \%$ & $13.5 \%$ & $9.1 \%$ & $30.5 \%$ & $35.9 \%$ & $35.7 \%$ & $27.3 \%$ & $0.0 \%$ & $35.5 \%$ \\
\hline$\%$ cases with Frozen & $39.3 \%$ & $31.3 \%$ & $18.1 \%$ & $13.2 \%$ & $31.3 \%$ & $36.5 \%$ & $33.8 \%$ & $19.4 \%$ & $16.2 \%$ & $32.8 \%$ & $35.7 \%$ & $34.4 \%$ & $15.3 \%$ & $0.0 \%$ & $34.0 \%$ \\
\hline Util Rate/Eggs & $28.3 \%$ & $27.0 \%$ & $21.8 \%$ & $35.4 \%$ & $26.7 \%$ & $28.7 \%$ & $27.5 \%$ & $24.9 \%$ & $23.2 \%$ & $27.6 \%$ & $28.1 \%$ & $26.9 \%$ & $24.3 \%$ & $40.0 \%$ & $27.5 \%$ \\
\hline Util Rate/2PN & $51.2 \%$ & $48.4 \%$ & $43.5 \%$ & $54.7 \%$ & $48.7 \%$ & $49.7 \%$ & $49.0 \%$ & $47.4 \%$ & $48.6 \%$ & $49.2 \%$ & $48.4 \%$ & $51.0 \%$ & $46.5 \%$ & $40.0 \%$ & $49.0 \%$ \\
\hline Util Rate/ICSII 2PN & $57.9 \%$ & $59.3 \%$ & $47.5 \%$ & $61.7 \%$ & $56.5 \%$ & $59.3 \%$ & $56.3 \%$ & $53.9 \%$ & $50.5 \%$ & $57.4 \%$ & $56.5 \%$ & $58.1 \%$ & $54.0 \%$ & $40.0 \%$ & $56.7 \%$ \\
\hline Preg Prod/Init Cycle & $67.5 \%$ & $47.9 \%$ & $18.4 \%$ & $4.0 \%$ & $42.7 \%$ & $65.5 \%$ & $44.5 \%$ & $20.0 \%$ & $6.7 \%$ & $46.5 \%$ & $69.0 \%$ & $49.6 \%$ & $13.7 \%$ & $0.0 \%$ & $55.7 \%$ \\
\hline Preg Prod/OPU & $76.4 \%$ & $52.7 \%$ & $21.6 \%$ & $4.3 \%$ & $48.2 \%$ & $71.7 \%$ & $49.4 \%$ & $22.4 \%$ & $8.7 \%$ & $51.9 \%$ & $76.0 \%$ & $53.2 \%$ & $15.9 \%$ & $0.0 \%$ & $61.5 \%$ \\
\hline Miscarriage Rate/Preg & $4.9 \%$ & $13.0 \%$ & $28.6 \%$ & $0.0 \%$ & $11.0 \%$ & $12.3 \%$ & $4.7 \%$ & $6.1 \%$ & $3.3 \%$ & $6.3 \%$ & $7.6 \%$ & $10.9 \%$ & $3.9 \%$ & $0.0 \%$ & $6.7 \%$ \\
\hline L/B Prod/Init Cycle & $54.2 \%$ & $38.2 \%$ & $10.5 \%$ & $4.0 \%$ & $33.0 \%$ & $50.1 \%$ & $31.1 \%$ & $11.1 \%$ & $5.0 \%$ & $33.9 \%$ & $57.2 \%$ & $37.8 \%$ & $5.9 \%$ & $0.0 \%$ & $44.5 \%$ \\
\hline L/B Prod /OPU & $61.3 \%$ & $42.0 \%$ & $12.4 \%$ & $4.3 \%$ & $37.3 \%$ & $54.9 \%$ & $34.6 \%$ & $12.4 \%$ & $6.5 \%$ & $37.8 \%$ & $63.0 \%$ & $40.5 \%$ & $6.8 \%$ & $0.0 \%$ & $49.2 \%$ \\
\hline
\end{tabular}

\subsection{Distributions}

The distribution of IGF-1 levels for the quartiles (lowest, IQR and highest) among the 1747 IVF treatment cycles (from 1035 women) is shown in Figure 2. There are several significant findings to note - firstly IGF-1 levels decline with age at each quartile; secondly the proportion of women with the highest IGF-1 levels are the younger; and thirdly the proportion of women aged $\geq 40$ years in the lowest IGF-1 quartile is significantly higher $(139 / 429 ; 32.4 \%)$, than in the highest quartile $(53 / 429 ; 12.4 \%)(p=0.0001)$.

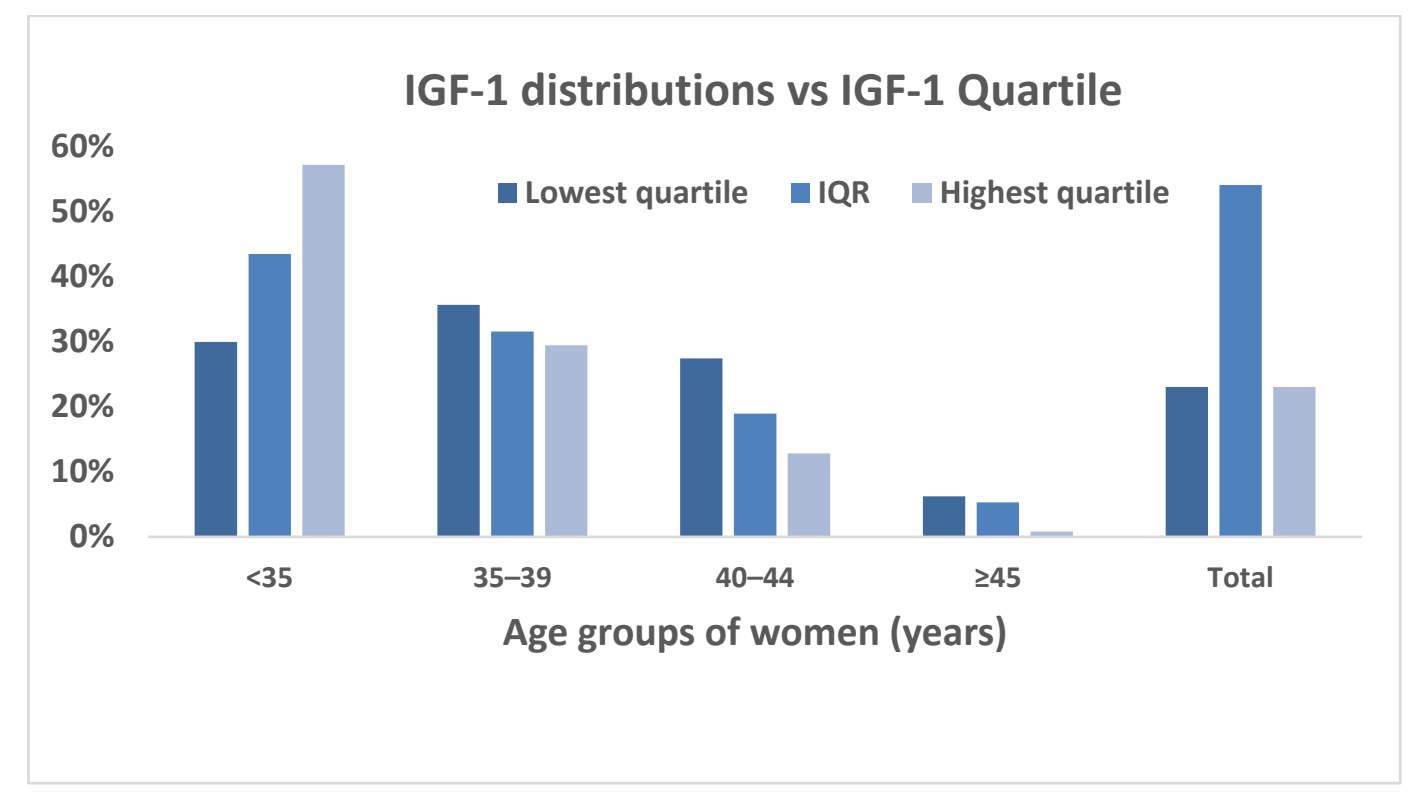

Figure 2 Shows the IGF-1 quartile distributions across 4 age groups of women undertaking IVF. The IGF-1 levels were undertaken on 1035 women in a preliminary Assessment Cycle (around Day-5) prior to initiating 1747 IVF cycles.

With respect to IGFBP-3 levels among the women with initiated cycles, there were more women aged $\geq 40$ years in the lowest quartile $(108 / 426 ; 25.4 \%$ cycles) when compared to the highest quartile $(82 / 426 ; 19.2 \%)$ but this difference was of borderline significance $(\mathrm{p}=0.03)$. With respect to the IGF ratio, there were more women $\geq 40$ years in the highest quartile $169 / 430 ; 39.3 \%$ cycles) when compared to lowest quartile $(68 / 430 ; 15.8 \%$ cycles) and this difference was highly significant $(\mathrm{p}<0.0001)$. Given that the IGF ratio is highly influenced by the IGF-1 level from the formula IGFBP-3/ IGF-1, it is likely that the main significant differences with respect to the case distributions for women above or below age 40 years, relates to the IGF-1 levels, with IGFBP-3 playing no significant role. 
Table 4a Tabulates the raw data for the IGFBP-3 quartiles for the key outcome measures of the 1035 women who initiated 1747 IVF treatment cycles.

\begin{tabular}{|c|c|c|c|c|c|c|c|c|c|c|c|c|c|c|c|}
\hline IGFBP-3 levels & & $<1$ & $9 \mathrm{nmol}$ & & & & 149 & $186 \mathrm{nmo}$ & & & & $>1$ & $6 \mathrm{nmol} /$ & & \\
\hline Age Groups years & $<35$ & 35-39 & $40-44$ & $\geq 45$ & Total & $<35$ & 35-39 & $40-44$ & $\geq 45$ & Total & $<35$ & $35-39$ & $40-44$ & $\geq 45$ & Total \\
\hline Initiated Cycles & 155 & 141 & 78 & 30 & 404 & 401 & 297 & 198 & 38 & 934 & 200 & 121 & 64 & 18 & 403 \\
\hline Patients Represented & 105 & 86 & 37 & 16 & 244 & 260 & 159 & 97 & 19 & 535 & 138 & 72 & 32 & 10 & 252 \\
\hline Cancelled cycles & 15 & 17 & 6 & 9 & 47 & 42 & 26 & 30 & 5 & 103 & 13 & 8 & 7 & 3 & 31 \\
\hline \#OPU & 140 & 124 & 72 & 21 & 357 & 359 & 271 & 168 & 33 & 831 & 187 & 113 & 57 & 15 & 372 \\
\hline Oocytes Collected & 1537 & 1159 & 509 & 80 & 3285 & 4174 & 2566 & 1261 & 178 & 8179 & 2242 & 1104 & 366 & 45 & 3757 \\
\hline Minimum Oocytes/OPU & 1 & 1 & 1 & 1 & 1 & 1 & 1 & 1 & 1 & 1 & 1 & 1 & 1 & 1 & 1 \\
\hline Maximum Oocytes/OPU & 35 & 26 & 20 & 13 & 35 & 36 & 39 & 30 & 16 & 39 & 36 & 24 & 18 & 11 & 36 \\
\hline Std Deviation (oocytes) & 6.4 & 5 & 4 & 3 & 19.1 & 6.4 & 6 & 5 & 4 & 22 & 6.5 & 5 & 4 & 3 & 19.3 \\
\hline Increased Monitoring & 51 & 30 & 11 & 1 & 93 & 132 & 72 & 28 & 4 & 236 & 71 & 29 & 7 & 1 & 108 \\
\hline Oocytes Injected & 965 & 737 & 346 & 44 & 2092 & 2676 & 1671 & 821 & 136 & 5304 & 1408 & 723 & 281 & 33 & 2445 \\
\hline$\# 2 \mathrm{PN}$ & 816 & 652 & 255 & 33 & 1756 & 2392 & 1419 & 656 & 99 & 4566 & 1368 & 595 & 195 & 27 & 2185 \\
\hline \#2PN (ICSI Only) & 725 & 535 & 241 & 33 & 1534 & 2053 & 1241 & 553 & 97 & 3944 & 1113 & 511 & 189 & 23 & 1836 \\
\hline \#Freeze-all embryos & 17 & 10 & 6 & 0 & 33 & 49 & 45 & 20 & 5 & 119 & 20 & 12 & 7 & 6 & 45 \\
\hline \#ET cycles & 113 & 108 & 58 & 13 & 292 & 295 & 194 & 127 & 23 & 639 & 159 & 87 & 42 & 4 & 292 \\
\hline \#Fresh embryos transf. & 118 & 114 & 74 & 18 & 324 & 307 & 224 & 176 & 34 & 741 & 163 & 89 & 58 & 6 & 316 \\
\hline \#Embryos Frozen (f) & 295 & 213 & 40 & 0 & 548 & 903 & 477 & 139 & 15 & 1534 & 475 & 196 & 24 & 8 & 703 \\
\hline \#FET Cycles (f) & 109 & 77 & 27 & 0 & 213 & 333 & 201 & 71 & 9 & 614 & 140 & 80 & 16 & 7 & 243 \\
\hline \#FET embryos transf. & 108 & 81 & 29 & 0 & 218 & 344 & 209 & 73 & 12 & 638 & 139 & 82 & 18 & 8 & 247 \\
\hline Total ETs F/f & 226 & 195 & 103 & 18 & 542 & 651 & 433 & 249 & 46 & 1379 & 302 & 171 & 76 & 14 & 563 \\
\hline Total Embryos Utilized & 413 & 327 & 114 & 18 & 872 & 1210 & 701 & 315 & 49 & 2275 & 638 & 285 & 82 & 14 & 1019 \\
\hline \#Pregs IVF only & 41 & 37 & 8 & 3 & 89 & 127 & 48 & 18 & 1 & 194 & 65 & 30 & 8 & 0 & 103 \\
\hline \#Pregnancies FET & 56 & 31 & 8 & 0 & 95 & 147 & 77 & 17 & 1 & 242 & 70 & 37 & 4 & 0 & 111 \\
\hline \#Pregs $\mathrm{F} / \mathrm{f}$ & 97 & 68 & 16 & 3 & 184 & 274 & 125 & 35 & 2 & 436 & 135 & 67 & 12 & 0 & 214 \\
\hline \#Miscarriages & 10 & 9 & 5 & 1 & 25 & 23 & 17 & 9 & 0 & 49 & 16 & 9 & 5 & 1 & 31 \\
\hline \#LiveBirths Fresh & 34 & 31 & 2 & 2 & 69 & 109 & 31 & 11 & 1 & 152 & 54 & 21 & 2 & 0 & 77 \\
\hline \#Livebirths FET & 46 & 25 & 4 & 0 & 75 & 108 & 57 & 12 & 1 & 178 & 50 & 28 & 3 & 0 & 81 \\
\hline \#LiveBirths F/f & 80 & 56 & 6 & 2 & 144 & 217 & 88 & 23 & 2 & 330 & 104 & 49 & 5 & 0 & 158 \\
\hline
\end{tabular}

Table $4 \mathbf{b}$ Tabulates the data for the IGFBP-3 quartiles as rates for the key outcome measures of the 1035 women who initiated 1747 IVF treatment cycles.

\begin{tabular}{|c|c|c|c|c|c|c|c|c|c|c|c|c|c|c|c|}
\hline IGFBP-3 levels & \multicolumn{5}{|c|}{$<149 \mathrm{nmol} / \mathrm{L}$} & \multicolumn{5}{|c|}{$149-186 \mathrm{nmol} / \mathrm{L}$} & \multicolumn{5}{|c|}{$>186 \mathrm{nmol} / \mathrm{L}$} \\
\hline Age Groups years & $<35$ & 35-39 & 40-44 & $\geq 45$ & Total & $<35$ & 35-39 & $40-44$ & $\geq 45$ & Total & $<35$ & 35-39 & $40-44$ & $\geq 45$ & Total \\
\hline Cancellation Rate & $9.7 \%$ & $12.1 \%$ & $7.7 \%$ & $30.0 \%$ & $11.6 \%$ & $10.5 \%$ & $8.8 \%$ & $15.2 \%$ & $13.2 \%$ & $11.0 \%$ & $6.5 \%$ & $6.6 \%$ & $10.9 \%$ & $16.7 \%$ & $7.7 \%$ \\
\hline Oocytes/OPU & 11.0 & 9.3 & 7.1 & 3.8 & 9.2 & 11.6 & 9.5 & 7.5 & 5.4 & 9.8 & 12.0 & 9.8 & 6.4 & 3.0 & 10.1 \\
\hline Fert Rate $(\mathrm{IVF} \pm \mathrm{IC}$ & $3.1 \%$ & $56.3 \%$ & $0.1 \%$ & $41.3 \%$ & $3.5 \%$ & $57.3 \%$ & $55.3 \%$ & $52.0 \%$ & $55.6 \%$ & $55.8 \%$ & $61.0 \%$ & $53.9 \%$ & $53.3 \%$ & $60.0 \%$ & $58.2 \%$ \\
\hline Fert Rate (ICSI) & $.2 \%$ & $46.2 \%$ & $47.3 \%$ & $41.3 \%$ & $46.7 \%$ & $49.2 \%$ & $48.4 \%$ & $43.9 \%$ & $54.5 \%$ & $48.2 \%$ & $49.6 \%$ & $46.3 \%$ & $51.6 \%$ & $51.1 \%$ & $48.9 \%$ \\
\hline Preg I & $.5 \%$ & $26.2 \%$ & $10.3 \%$ & $10.0 \%$ & $22.0 \%$ & $31.7 \%$ & $16.2 \%$ & $9.1 \%$ & $2.6 \%$ & $20.8 \%$ & $32.5 \%$ & $4.8 \%$ & $12.5 \%$ & $0.0 \%$ & $5.6 \%$ \\
\hline Preg F & $1.4 \%$ & $40.3 \%$ & $29.6 \%$ & $0.0 \%$ & $44.6 \%$ & $44.1 \%$ & $38.3 \%$ & $23.9 \%$ & $11.1 \%$ & $39.4 \%$ & $50.0 \%$ & $46.3 \%$ & $25.0 \%$ & $0.0 \%$ & $5.7 \%$ \\
\hline Livebirth R & $1.9 \%$ & $22.0 \%$ & $2.6 \%$ & $6.7 \%$ & $17.1 \%$ & $27.2 \%$ & $10.4 \%$ & $5.6 \%$ & $2.6 \%$ & $16.3 \%$ & $27.0 \%$ & $17.4 \%$ & $3.1 \%$ & $0.0 \%$ & $19.1 \%$ \\
\hline Livebirth R & $42.2 \%$ & $32.5 \%$ & $14.8 \%$ & $0.0 \%$ & $35.2 \%$ & $32.4 \%$ & $28.4 \%$ & $16.9 \%$ & $11.1 \%$ & $29.0 \%$ & $35.7 \%$ & $35.0 \%$ & $18.8 \%$ & $0.0 \%$ & $33.3 \%$ \\
\hline$\% \mathrm{cc}$ & $36.2 \%$ & $32.7 \%$ & $15.7 \%$ & $0.0 \%$ & $31.2 \%$ & $37.8 \%$ & $33.6 \%$ & $21.2 \%$ & $15.2 \%$ & $33.6 \%$ & $34.7 \%$ & $32.9 \%$ & $12.3 \%$ & $29.6 \%$ & $32.2 \%$ \\
\hline Util Rate/Eggs & $26.9 \%$ & $28.2 \%$ & $22.4 \%$ & $22.5 \%$ & $26.5 \%$ & $29.0 \%$ & $27.3 \%$ & $25.0 \%$ & $27.5 \%$ & $27.8 \%$ & $28.5 \%$ & $25.8 \%$ & $22.4 \%$ & $31.1 \%$ & $27.1 \%$ \\
\hline Util Rate/2PN & $50.6 \%$ & $50.2 \%$ & $44.7 \%$ & $54.5 \%$ & $49.7 \%$ & $50.6 \%$ & $49.4 \%$ & $48.0 \%$ & $49.5 \%$ & $49.8 \%$ & $46.6 \%$ & $47.9 \%$ & $42.1 \%$ & $51.9 \%$ & $46.6 \%$ \\
\hline Util R & $.0 \%$ & $61.1 \%$ & $47.3 \%$ & $54.5 \%$ & $56.8 \%$ & $58.9 \%$ & $56.5 \%$ & $57.0 \%$ & $50.5 \%$ & $57.7 \%$ & $57.3 \%$ & $55.8 \%$ & $43.4 \%$ & $60.9 \%$ & $55.5 \%$ \\
\hline Preg I & $62.6 \%$ & $48.2 \%$ & $20.5 \%$ & $10.0 \%$ & $45.5 \%$ & $68.3 \%$ & $42.1 \%$ & $17.7 \%$ & $5.3 \%$ & $46.7 \%$ & $67.5 \%$ & $55.4 \%$ & $18.8 \%$ & $0.0 \%$ & $53.1 \%$ \\
\hline Preg Prod /OPU & $69.3 \%$ & $54.8 \%$ & $22.2 \%$ & $14.3 \%$ & $51.5 \%$ & $76.3 \%$ & $46.1 \%$ & $20.8 \%$ & $6.1 \%$ & $52.5 \%$ & $72.2 \%$ & $59.3 \%$ & $21.1 \%$ & $0.0 \%$ & $57.5 \%$ \\
\hline Miscarriage Rate/ & $10.3 \%$ & $13.2 \%$ & $31.3 \%$ & $33.3 \%$ & $13.6 \%$ & $8.4 \%$ & $5.7 \%$ & $4.5 \%$ & $0.0 \%$ & $5.2 \%$ & $11.9 \%$ & $7.4 \%$ & $7.8 \%$ & $5.6 \%$ & $7.7 \%$ \\
\hline L/B Prod/Init Cy & $51.6 \%$ & $39.7 \%$ & $7.7 \%$ & $6.7 \%$ & $35.6 \%$ & $54.1 \%$ & $29.6 \%$ & $11.6 \%$ & $5.3 \%$ & $35.3 \%$ & $52.0 \%$ & $40.5 \%$ & $7.8 \%$ & $0.0 \%$ & $39.2 \%$ \\
\hline L/B Prod /OPU & $57.1 \%$ & $45.2 \%$ & $8.3 \%$ & $9.5 \%$ & $40.3 \%$ & $60.4 \%$ & $32.5 \%$ & $13.7 \%$ & $6.1 \%$ & $39.7 \%$ & $55.6 \%$ & $43.4 \%$ & $8.8 \%$ & $0.0 \%$ & $42.5 \%$ \\
\hline
\end{tabular}


Table 5a Tabulates the raw data for the IGF ratio (IGFBP-3/IGF-1) quartiles for the key outcome measures of the 1035 women who initiated 1747 IVF treatment cycles.

\begin{tabular}{|c|c|c|c|c|c|c|c|c|c|c|c|c|c|c|c|}
\hline IGF ratios & & & $<5.7$ & & & & & $5.7-7.8$ & & & & & $>7.8$ & & \\
\hline Age Groups years & $<35$ & 35-39 & $40-44$ & $\geq 45$ & Total & $<35$ & 35-39 & $40-44$ & $\geq 45$ & Total & $<35$ & 35-39 & $40-44$ & $\geq 45$ & Total \\
\hline Initiated Cycles & 214 & 137 & 50 & 18 & 419 & 417 & 286 & 153 & 40 & 896 & 132 & 136 & 139 & 30 & 437 \\
\hline Patients Represented & 147 & 67 & 25 & 10 & 249 & 271 & 170 & 78 & 19 & 538 & 90 & 82 & 64 & 18 & 254 \\
\hline Cancelled cycles & 21 & 18 & 8 & 8 & 55 & 36 & 18 & 11 & 7 & 72 & 14 & 13 & 23 & 3 & 53 \\
\hline \#OPU & 193 & 119 & 42 & 10 & 364 & 381 & 268 & 142 & 33 & 824 & 118 & 123 & 116 & 27 & 384 \\
\hline Oocytes Collected & 2221 & 1088 & 284 & 32 & 3625 & 4350 & 2598 & 1032 & 184 & 8164 & 1453 & 1161 & 837 & 90 & 3541 \\
\hline Minimum Oocytes/OPU & 2 & 1 & 1 & 1 & 1 & 1 & 1 & 1 & 1 & 1 & 1 & 1 & 1 & 1 & 1 \\
\hline Maximum Oocytes/OPU & 36 & 31 & 23 & 7 & 36 & 36 & 39 & 30 & 16 & 39 & 36 & 29 & 23 & 13 & 36 \\
\hline Std Deviation (oocytes) & 5.3 & 6 & 5 & 2 & 18.2 & 6.7 & 6 & 5 & 4 & 21.7 & 7.1 & 6 & 5 & 3 & 20.4 \\
\hline Increased Monitoring & 68 & 32 & 7 & 0 & 107 & 140 & 71 & 21 & 5 & 237 & 49 & 29 & 19 & 1 & 98 \\
\hline Oocytes Injected & 1393 & 700 & 176 & 16 & 2285 & 2757 & 1691 & 691 & 122 & 5261 & 947 & 761 & 585 & 77 & 2370 \\
\hline \#2PN & 1276 & 593 & 146 & 14 & 2029 & 2452 & 1433 & 538 & 84 & 4507 & 891 & 658 & 430 & 62 & 2041 \\
\hline \#2PN (ICSI Only) & 1093 & 511 & 124 & 14 & 1742 & 2084 & 1235 & 483 & 84 & 3886 & 752 & 559 & 381 & 56 & 1748 \\
\hline \#Freeze-all embryos & 26 & 12 & 3 & 0 & 41 & 44 & 41 & 15 & 5 & 105 & 17 & 15 & 16 & 6 & 54 \\
\hline \#ET cycles & 162 & 92 & 32 & 6 & 292 & 317 & 203 & 115 & 19 & 654 & 93 & 97 & 79 & 16 & 285 \\
\hline \#Fresh embryos transf. & 171 & 101 & 43 & 9 & 324 & 329 & 228 & 156 & 24 & 737 & 93 & 101 & 108 & 26 & 328 \\
\hline \#Embryos Frozen (f) & 460 & 192 & 29 & 0 & 681 & 915 & 483 & 100 & 14 & 1512 & 320 & 218 & 78 & 9 & 625 \\
\hline \#FET Cycles (f) & 157 & 89 & 18 & 0 & 264 & 306 & 198 & 57 & 9 & 570 & 123 & 75 & 42 & 7 & 247 \\
\hline \#FET embryos transf. & 157 & 93 & 18 & 0 & 268 & 315 & 207 & 61 & 11 & 594 & 123 & 76 & 44 & 9 & 252 \\
\hline Total ETs F/f & 328 & 194 & 61 & 9 & 592 & 644 & 435 & 217 & 35 & 1331 & 216 & 177 & 152 & 35 & 580 \\
\hline Total Embryos Utilized & 631 & 293 & 72 & 9 & 1005 & 1244 & 711 & 256 & 38 & 2249 & 413 & 319 & 186 & 35 & 953 \\
\hline \#Pregs IVF only & 63 & 25 & 1 & 0 & 89 & 128 & 60 & 17 & 3 & 208 & 45 & 31 & 15 & 1 & 92 \\
\hline \#Pregnancies FET & 79 & 32 & 6 & 0 & 117 & 142 & 82 & 14 & 1 & 239 & 56 & 32 & 10 & 0 & 98 \\
\hline \#Pregs F/f & 142 & 57 & 7 & 0 & 206 & 270 & 142 & 31 & 4 & 447 & 101 & 63 & 25 & 1 & 190 \\
\hline \#Miscarriages & 16 & 16 & 1 & 0 & 33 & 27 & 15 & 11 & 2 & 55 & 7 & 5 & 6 & 0 & 18 \\
\hline \#LiveBirths Fresh & 52 & 14 & 0 & 0 & 66 & 107 & 45 & 8 & 2 & 162 & 39 & 24 & 7 & 1 & 71 \\
\hline \#Livebirths FET & 61 & 28 & 5 & 0 & 94 & 110 & 59 & 7 & 1 & 177 & 37 & 24 & 8 & 0 & 69 \\
\hline \#LiveBirths F/f & 113 & 42 & 5 & 0 & 160 & 217 & 104 & 15 & 3 & 339 & 76 & 48 & 15 & 1 & 140 \\
\hline
\end{tabular}

Table 5b Tabulates the data for the IGF ratio (IGFBP-3/IGF-1) quartiles as rates for the key outcome measures of the 1035 women who initiated 1747 IVF treatment cycles.

\begin{tabular}{|c|c|c|c|c|c|c|c|c|c|c|c|c|c|c|c|}
\hline IGF ratios & \multicolumn{5}{|c|}{$<5.7$} & \multicolumn{5}{|c|}{$5.7-7.8$} & \multicolumn{5}{|c|}{$>7.8$} \\
\hline Age Groups years & $<35$ & $35-39$ & $40-44$ & $\geq 45$ & Total & $<35$ & $35-39$ & $40-44$ & $\geq 45$ & Total & $<35$ & $35-39$ & $40-44$ & $\geq 45$ & Total \\
\hline Cancellation Rate & $9.8 \%$ & $13.1 \%$ & $16.0 \%$ & $44.4 \%$ & $13.1 \%$ & $8.6 \%$ & $6.3 \%$ & $7.2 \%$ & $17.5 \%$ & $8.0 \%$ & $10.6 \%$ & $9.6 \%$ & $16.5 \%$ & $10.0 \%$ & $12.1 \%$ \\
\hline Oocytes/OPU & 11.5 & 9.1 & 6.8 & 3.2 & 10.0 & 11.4 & 9.7 & 7.3 & 5.6 & 9.9 & 12.3 & 9.4 & 7.2 & 3.3 & 9.2 \\
\hline Fert Rate (IVF \pm ICSI) & $57.5 \%$ & $54.5 \%$ & $51.4 \%$ & $43.8 \%$ & $56.0 \%$ & $56.4 \%$ & $55.2 \%$ & $52.1 \%$ & $45.7 \%$ & $55.2 \%$ & $61.3 \%$ & $56.7 \%$ & $51.4 \%$ & $68.9 \%$ & $57.6 \%$ \\
\hline (ICSI) & $49.2 \%$ & $47.0 \%$ & $43.7 \%$ & $43.8 \%$ & $48.1 \%$ & $47.9 \%$ & $47.5 \%$ & $46.8 \%$ & $45.7 \%$ & $47.6 \%$ & $51.8 \%$ & $48.1 \%$ & $45.5 \%$ & $62.2 \%$ & $49.4 \%$ \\
\hline Preg R & $29.4 \%$ & $18.2 \%$ & $2.0 \%$ & $0.0 \%$ & $21.2 \%$ & $30.7 \%$ & $21.0 \%$ & $11.1 \%$ & $7.5 \%$ & $23.2 \%$ & $34.1 \%$ & $22.8 \%$ & $10.8 \%$ & $3.3 \%$ & $21.1 \%$ \\
\hline Preg R & $50.3 \%$ & $36.0 \%$ & $33.3 \%$ & $0.0 \%$ & $44.3 \%$ & $46.4 \%$ & $41.4 \%$ & $24.6 \%$ & $11.1 \%$ & $41.9 \%$ & $45.5 \%$ & $42.7 \%$ & $23.8 \%$ & $0.0 \%$ & $39.7 \%$ \\
\hline Liveb & $24.3 \%$ & $10.2 \%$ & $0.0 \%$ & $0.0 \%$ & $15.8 \%$ & $25.7 \%$ & $15.7 \%$ & $5.2 \%$ & $5.0 \%$ & $18.1 \%$ & $29.5 \%$ & $17.6 \%$ & $5.0 \%$ & $3.3 \%$ & $16.2 \%$ \\
\hline Livet & $38.9 \%$ & $31.5 \%$ & $27.8 \%$ & $0.0 \%$ & $35.6 \%$ & $35.9 \%$ & $29.8 \%$ & $12.3 \%$ & $11.1 \%$ & $31.1 \%$ & $30.1 \%$ & $32.0 \%$ & $19.0 \%$ & $0.0 \%$ & $27.9 \%$ \\
\hline$\%$ cas & $36.1 \%$ & $32.4 \%$ & $19.9 \%$ & $0.0 \%$ & $33.6 \%$ & $37.3 \%$ & $33.7 \%$ & $18.6 \%$ & $16.7 \%$ & $33.5 \%$ & $35.9 \%$ & $33.1 \%$ & $18.1 \%$ & $14.5 \%$ & $30.6 \%$ \\
\hline Util R & $28.4 \%$ & $26.9 \%$ & $25.4 \%$ & $28.1 \%$ & $27.7 \%$ & $28.6 \%$ & $27.4 \%$ & $24.8 \%$ & $20.7 \%$ & $27.5 \%$ & $28.4 \%$ & $27.5 \%$ & $22.2 \%$ & $38.9 \%$ & $26.9 \%$ \\
\hline Util R & $49.5 \%$ & $49.4 \%$ & $49.3 \%$ & $64.3 \%$ & $49.5 \%$ & $50.7 \%$ & $49.6 \%$ & $47.6 \%$ & $45.2 \%$ & $49.9 \%$ & $46.4 \%$ & $48.5 \%$ & $43.3 \%$ & $56.5 \%$ & $46.7 \%$ \\
\hline Util R & $57.7 \%$ & $57.3 \%$ & $58.1 \%$ & $64.3 \%$ & $57.7 \%$ & $59.7 \%$ & $57.6 \%$ & $53.0 \%$ & $45.2 \%$ & $57.9 \%$ & $54.9 \%$ & $57.1 \%$ & $48.8 \%$ & $62.5 \%$ & $54.5 \%$ \\
\hline Preg Prod/Init Cycle & $66.4 \%$ & $41.6 \%$ & $14.0 \%$ & $0.0 \%$ & $49.2 \%$ & $64.7 \%$ & $49.7 \%$ & $20.3 \%$ & $10.0 \%$ & $49.9 \%$ & $76.5 \%$ & $46.3 \%$ & $18.0 \%$ & $3.3 \%$ & $43.5 \%$ \\
\hline Preg Prod /OPU & $73.6 \%$ & $47.9 \%$ & $16.7 \%$ & $0.0 \%$ & $56.6 \%$ & $70.9 \%$ & $53.0 \%$ & $21.8 \%$ & $12.1 \%$ & $54.2 \%$ & $85.6 \%$ & $51.2 \%$ & $21.6 \%$ & $3.7 \%$ & $49.5 \%$ \\
\hline Miscarriage Rate/Preg & $11.3 \%$ & $28.1 \%$ & $14.3 \%$ & $0.0 \%$ & $16.0 \%$ & $10.0 \%$ & $5.2 \%$ & $7.2 \%$ & $5.0 \%$ & $6.1 \%$ & $6.9 \%$ & $3.7 \%$ & $4.3 \%$ & $0.0 \%$ & $4.1 \%$ \\
\hline L/B Prod/Init Cycle & $52.8 \%$ & $30.7 \%$ & $10.0 \%$ & $0.0 \%$ & $38.2 \%$ & $52.0 \%$ & $36.4 \%$ & $9.8 \%$ & $7.5 \%$ & $37.8 \%$ & $57.6 \%$ & $35.3 \%$ & $10.8 \%$ & $3.3 \%$ & $32.0 \%$ \\
\hline L/B Prod/OPU & $58.5 \%$ & $35.3 \%$ & $11.9 \%$ & $0.0 \%$ & $44.0 \%$ & $57.0 \%$ & $38.8 \%$ & $10.6 \%$ & $9.1 \%$ & $41.1 \%$ & $64.4 \%$ & $39.0 \%$ & $12.9 \%$ & $3.7 \%$ & $36.5 \%$ \\
\hline
\end{tabular}

\subsection{Cancellations}

From 402 cycles initiated, there were 46 cancellations because of markedly poor responses within the lowest IGF-1 profile, but these numbers were not significantly higher than the 39 cancellations from 401 cycles in the highest IGF-1 quartile, neither the 98 cancellations from 944 cycles in the IQR. With respect to IGBP-3 levels, the cancellation rates between the lowest quartile (47/404) and the highest quartile (31/403) were not significantly different. Similarly, for the IGF ratio, the cancellation rates between the lowest quartile (55/419) were not significantly different from the highest quartile at 53/437.

\subsection{Oocyte numbers}

The oocyte numbers retrieved at OPU are shown in Figure 3 for the 1572 IVF cycles which reached that stage. Consistently, more oocytes were recovered from those women with the highest IGF-1 levels $(3781 / 362 ; \mathrm{mean} \mathrm{n}=10.4)$ than from those in the lowest quartile $(3176 / 356$; mean $n=8.9)$, for both initiated cycles as well as for those reaching the stage of OPU $(\mathrm{p}=0.01)$. With respect to those 2060 oocytes shown to be at the mature metaphase (M-II) stage, (from 
402 initiated cycles; $\mathrm{n}=5.1$ ) in the lowest IGF-1 quartile as opposed to 2401 MII oocytes (from 401 initiated cycles; $\mathrm{n}=$ $6.0)$ in the highest quartile and suitable for ICSI, the data was equally significant $(p=0.01)$.

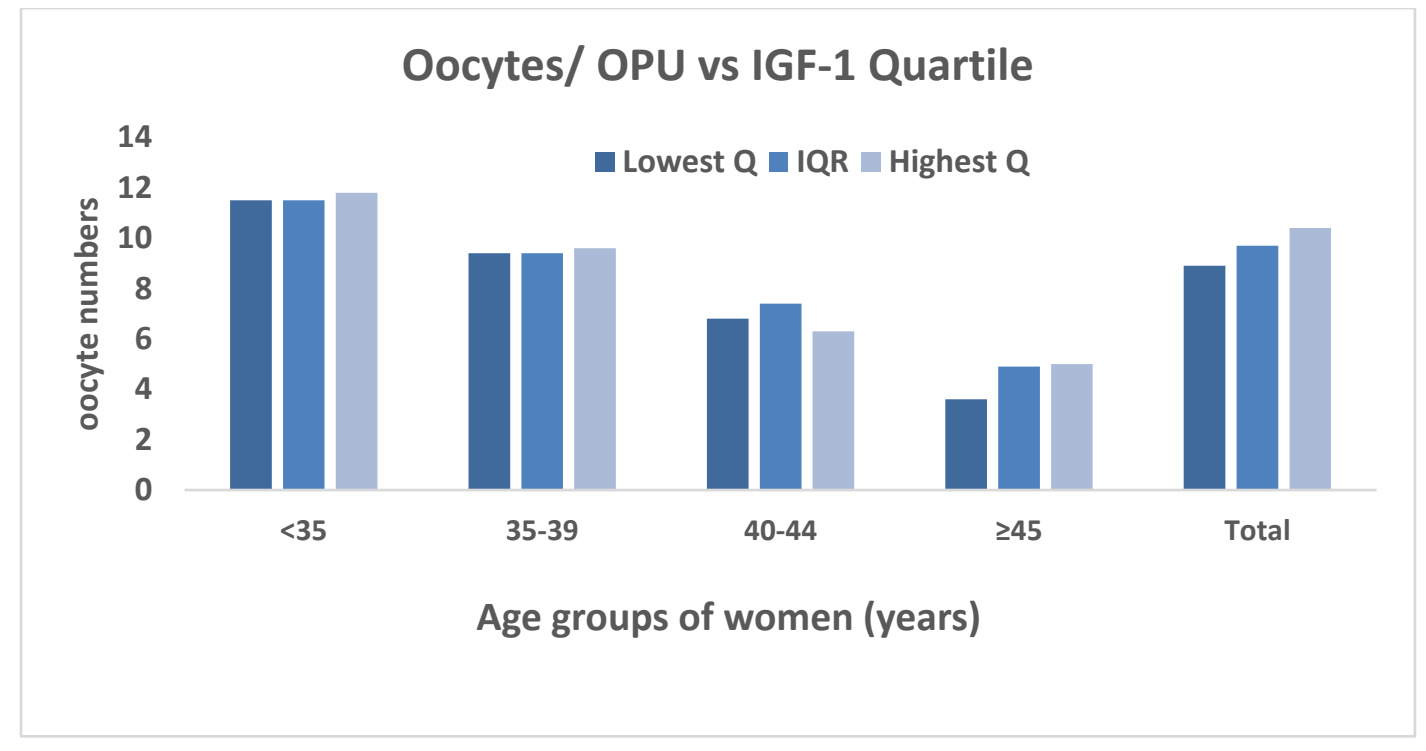

Figure 3 Displays the mean oocyte numbers retrieved at OPU across the four age groups according to the IGF-1 quartile distribution of the 1572 IVF cycles which reached the OPU stage.

With respect to the IGFBP-3 levels there were 3285 oocytes recovered from 357 OPUs (mean 9.2) arising from 404 initiated cycles (mean 8.1) in the lowest quartile and 3757 oocytes retrieved from 372 OPUs (mean 10.1) arising from 403 initiated cycles (mean 9.3) in the highest quartile. These values are not significantly different. Similarly, with respect to the quartiles for the IGF ratio, no significant differences in oocyte numbers were seen between the lowest quartile (3625 oocytes from 364 OPUs; mean 10.0) and the highest quartile (3541 oocytes from 384 OPUs; mean 9.2). Neither were there any significant differences with respect to cycles initiated being 419 for the lowest quartile; mean oocytes 8.7 and 437 for the highest quartile; mean oocytes 8.1 .

\subsection{Oocyte utilization}

The oocyte utilization rate is also significantly different among the IGF-1 quartiles. The number of embryos arising from oocytes in the lowest IGF-1 quartile was 1736 from 402 initiated cycles (4.3 embryos/cycle) being significantly lower than the 2134 embryos from 401 initiated cycles (5.3 embryos/cycle) in the highest quartile $(\mathrm{p}<0.001)$. The oocyte utilization rate was also significantly different when analyzing the number of embryos arising from IVF cycles which reached the OPU stage. For the lowest IGF-1 quartile the 1736 embryos arising from 356 OPUs (4.9 embryos/OPU) is significantly lower than the 2134 embryos arising from the 362 OPU procedures (5.9 embryos/OPU) in the highest quartile $(\mathrm{p}=0.005)$.

With respect to the IGFBP-3 levels, there were no significant differences between the lowest quartile (26.5\%) and the highest quartile (27.1\%). Similarly, for the IGF ratios, there were no significant differences between the lowest quartile $(28 \%)$ and the highest (27\%) for oocyte utilization rates.

\subsection{Distribution of oocyte numbers}

The oocyte distributions were similar among the women across the IGF-1 quartiles ranging from 1 to 36 amongst the youngest women ( $<35$ years) in each quartile and equivalent ranges for each of the 4 age groupings (no significant differences). Similarly, no significant differences were seen among the individual age groups for the quartile distributions for either the IGFBP-3 levels or the IGF ratios.

\subsection{Embryo quality and utilization}

Furthermore, the quality of the embryos generated showed no differences in relation to IGF-1 quartiles. In the lowest quartile 846 of the 1736 embryos were selected to be utilized (48.7\%) compared with 1043 of the 2134 embryos $(48.9 \%)$ arising from the highest quartile. Similarly, there was no difference in embryo utilization rates for the quartile distributions of neither the IGFBP-3 levels nor the IGF ratios. 


\subsection{Freeze-all rates}

Associated with high egg numbers, women considered to be at risk of OHSS were managed by utilizing a "freeze-all embryos" policy as well as with increased monitoring. The freeze-all rates between the lowest IGF-1 quartile was 44 cases from 356 OPU procedures to a similar 42 cases from 356 in the highest quartile (not significant). In the lowest IGF-1 quartile the proportion of women with increased monitoring was 89 women from 356 OPU procedures whereas the proportion was 115 women from 362 OPU procedures in the highest quartile. This difference in increased monitoring rates, being greater in the higher IGF-1 quartile, was of marginal significance $(p=0.04)$. There was no difference in either the freeze-all rates or the increased monitoring rates for the quartile distributions of neither the IGFBP-3 levels nor the IGF ratios.

\subsection{Pregnancies}

Pregnancy productivity outcomes are shown per IVF cycle initiated (Figure 4) and per IVF cycle reaching the stage of OPU (Figure 5).

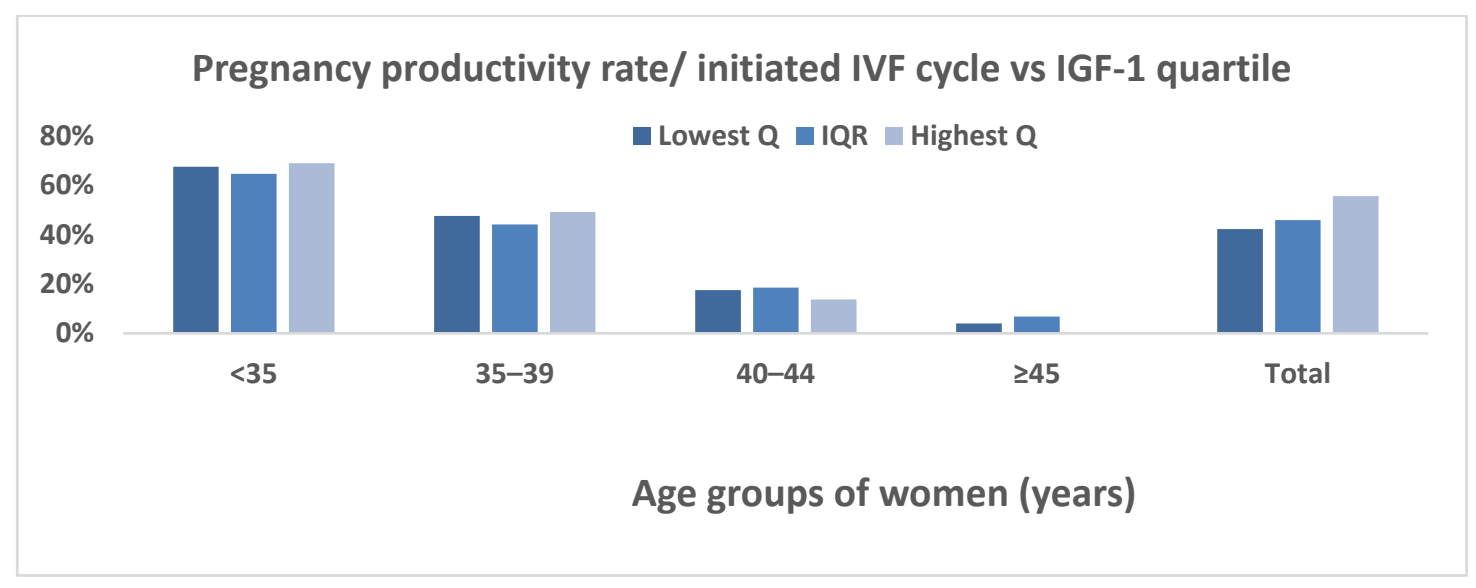

Figure 4 Depicts the pregnancy productivity rates across 4 age groups of women who initiated IVF treatment cycles. The pregnancies are shown according the quartiles of IGF-1 levels measured in a preliminary assessment cycle.

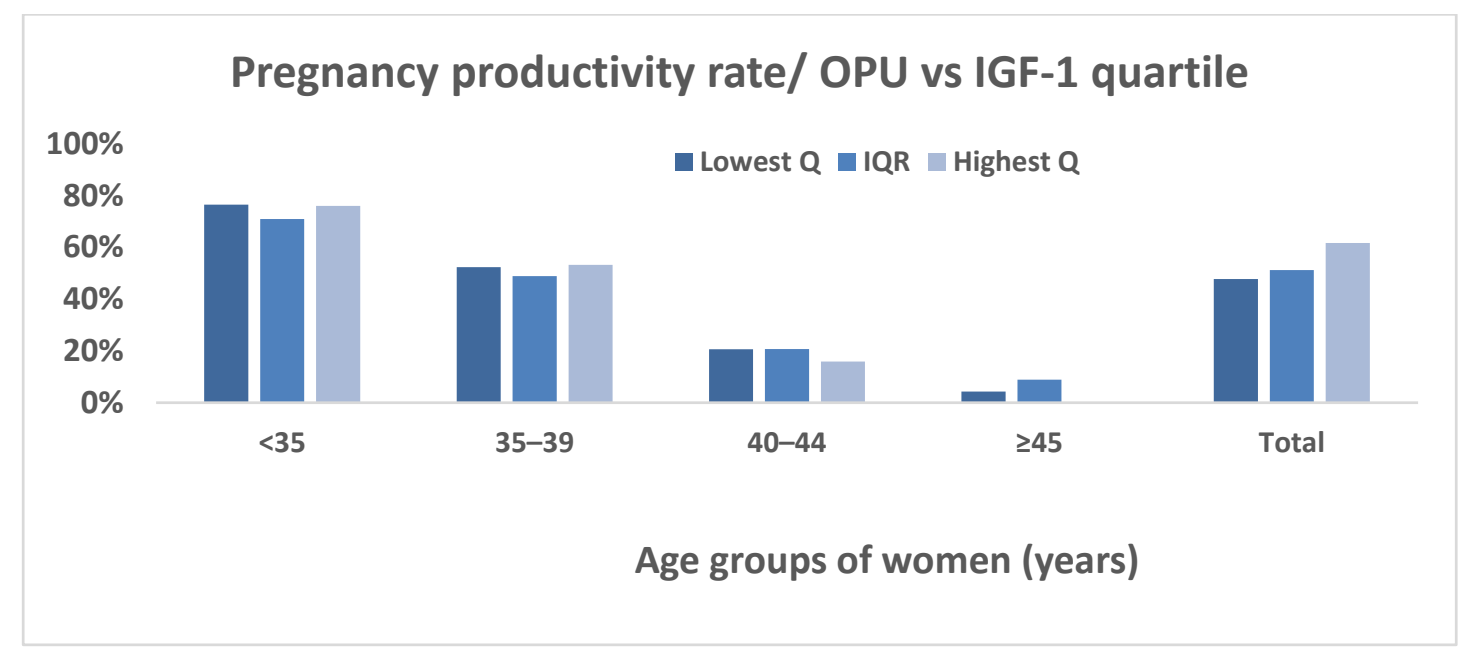

Figure 5 Depicts the pregnancy productivity rates across 4 age groups of women who reached the stage of OPU of their IVF treatment cycles. The pregnancies are shown according the quartiles of IGF-1 levels measured in a preliminary assessment cycle. 
The highest pregnancy productivity rates in both IVF cycles initiated and IVF cycles reaching the OPU stage, occurs in those women with IGF-1 levels in the highest quartile when compared to those with levels in the lowest quartile (per initiated cycles $\mathrm{p}<0.0002$; per OPU $\mathrm{p}<0.0002$ ). When the pregnancy productivity rates from the IGF-1 lowest quartile were compared with the IQR, the reduction was also significant (per initiated cycle $\mathrm{p}<0.002$; per 0 PU $p<0.001$ ). IGFBP3 quartiles showed no differences for pregnancy productivity. With respect to the IGF ratio quartiles, there was a marginal difference favoring the lowest quartile for pregnancy productivity (per initiated cycle $49 \%$ vs $43 \%$, p=0.04 $\mathrm{p}=0.04$; per OPU $57 \%$ vs $49 \% \mathrm{p}=0.04$ ).

\subsection{Miscarriage}

The miscarriage rates of clinically defined pregnancies across the IGF-1 quartiles ranged from $11 \%$ in the lowest quartile to $12 \%$ in the highest; such differences being not significant. The highest miscarriage rate (14\%) was recorded amongst those women in the IQR for IGF-1, but these differences across the quartiles were not significant. There were no differences in miscarriage rates related to the IGFBP-3 quartiles, nor for the quartiles of the IGF ratio.

\subsection{Live births}

The live birth productivity outcomes are shown per IVF cycle initiated (Figure 6) and per IVF cycle reaching the stage of OPU (Figure 7).

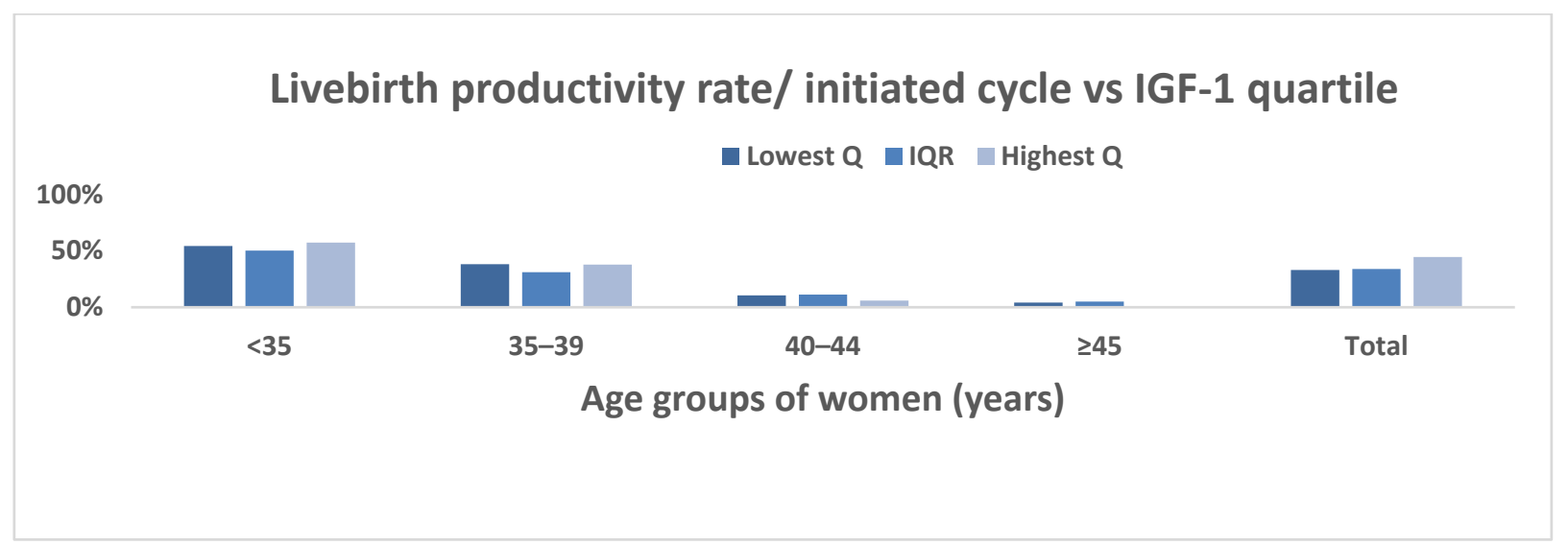

Figure 6 Depicts the live birth productivity rates across 4 age groups of women who initiated IVF treatment cycles. The pregnancies are shown according the quartiles of IGF-1 levels measured in a preliminary assessment cycle.

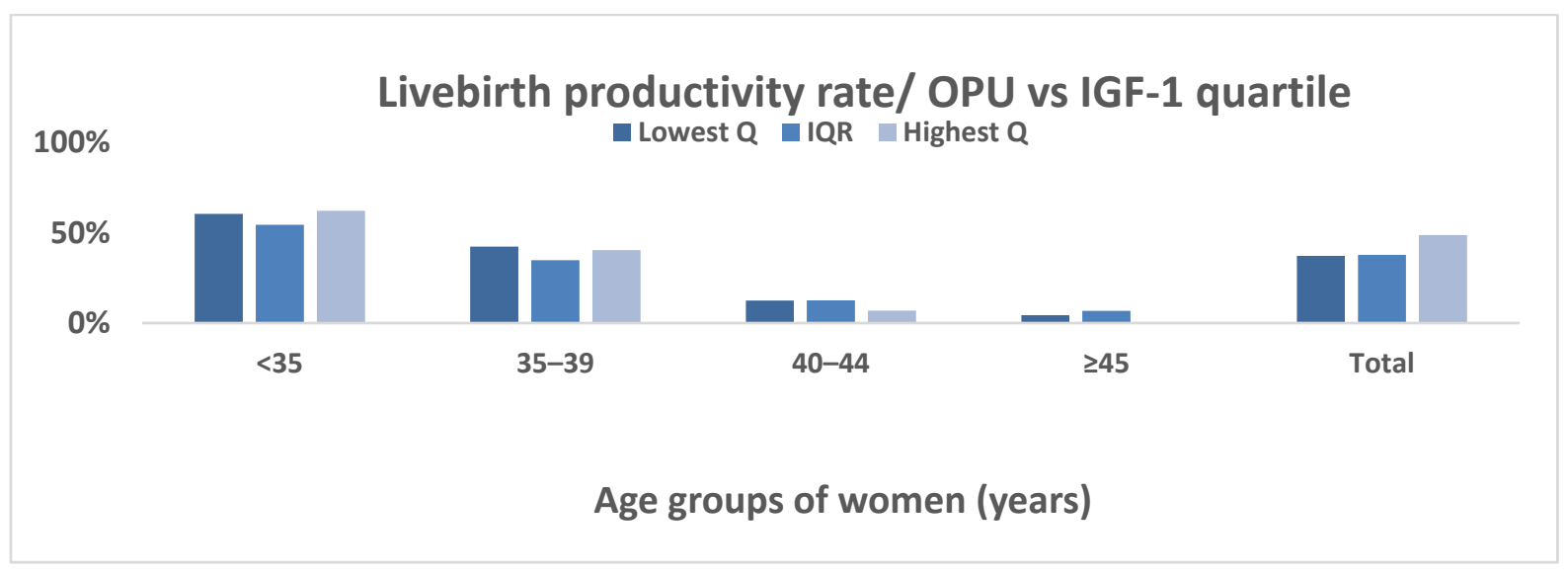

Figure 7 Depicts the live birth productivity rates across 4 age groups of women who reached the stage of OPU of their IVF treatment cycles. The pregnancies are shown according the quartiles of IGF-1 levels measured in a preliminary assessment cycle. 
The highest rates in both modes occurs in those women with IGF-1 levels in the highest quartile (44\% across all age groups per initiated cycle; $49 \%$ per OPU) when compared to the comparable rates (33\% per initiated cycle; $37 \%$ per OPU) for those women with levels in the lowest quartile (per initiated cycles $\mathrm{p}<0.002$; per OPU $\mathrm{p}<0.0002$ ). When the live birth productivity rates from the IGF-1 lowest quartile were compared with the IQR, the reduction was also highly significant (per initiated cycle $\mathrm{p}<0.002$; per OPU $\mathrm{p}<0.001$ ). IGFBP-3 quartiles showed no association with live birth outcomes, but the IGF ratio showed some borderline associations favouring the lowest quartile (livebirth productivity rate per initiated cycle $160 / 419 ; 38 \%$ vs $140 / 437 ; 32 \%$, p=0.06 and per OPU 160/364; 44\% vs 140/384; 36\%, p=0.04).

\section{Discussion}

In this fifth study, the relevance of each aspect of the IGF profile, namely IGF-1 levels, IGFBP-3 levels, and the IGF ratio, are examined with respect to clinical outcomes from ART treatments, specifically that of IVF \pm ICSI cycles. The cases examined are those of 1035 IVF-naïve women who had completed an Assessment Cycle which included full IGF profiling and who subsequently proceeded into 1747 IVF treatments during the period covering 2011 through 2019. Based on earlier studies undertaken on 1633 women (a larger group from which this group is derived), the IGF profile has been categorized into quartiles [Study1] and each of the clinical outcome parameters has been examined with particular reference to the highest and lowest quartiles.

The first parameter examined was that of the distribution of women across the quartiles, with reference to their age groups. This study confirmed our earlier report that IGF-1 levels decline across the quartiles with advancing age of the women, and the proportion of women with IGF-1 levels in the highest quartile comprised the younger, whilst those women $\geq 40$ years had IGF-1 levels mostly in the lowest quartile $(\mathrm{p}<0.0001)$. The IGF ratio also reflected this finding with the older women significantly more represented in the unfavorable higher quartile $(\mathrm{p}<0.0001)$. Given that IGFBP3 variations were of borderline significance and the IGF ratio (IGFBP-3/IGF-1) was therefore almost entirely reflective of the IGF-1 levels, we suggest that the IGF ratio does not contribute any further useful information. In fact, the further clinical outcomes showed no influence from IGFBP-3 levels, hence it appears only relevant to present the IGF-1 influence.

The number of oocytes recovered at OPU was significantly influenced by IGF-1 levels, with a higher mean number (10.4) associated with the highest IGF-1 quartile and conversely, the lower mean number (8.9) from those women with IGF-1 levels in the lowest quartile. This significant finding also pertained to the number of mature (M-II stage) oocytes recovered, being a mean of 6.0 per initiated cycle across all age groups, from those women in the highest IGF-1 quartile. This significant finding continued with both the oocyte utilization rate and the subsequent embryo utilization rates (5.9 embryos/OPU) also highly significantly related to the highest IGF-1 quartile.

The most important clinical findings were a highly significant pregnancy rate arising from the highest IGF-1 quartile, both for pregnancies from initiated cycles and per OPU. There was no variation in miscarriage rates from those pregnancies defined as "clinical stage", being at 11\% and 12\% in the lowest and highest quartiles respectively. This ensured that live birth rates were also highly significantly derived from those women with IGF-1 levels in the highest quartile, both for initiated IVF cycles and for those reaching OPU. The optimization of the pregnancy data applies the terms pregnancy productivity and live birth productivity rates, meaning the cumulative pregnancy outcomes from both fresh and frozen embryo transfers ensuing from a single OPU, and which can be as high as $63.0 \%$ for young women $(<35$ years) whose IGF-1 levels are in the highest quartile.

Whilst the mechanisms are not yet fully elucidated, it is clear that both GH and the IGF system play important roles in folliculogenesis [29,30]. The IGF system comprises IGF-1, IGF-2, type-1 IGF receptor (IGF-1R), IGF-2R and six IGF binding proteins that regulate IGF bioavailability; of which IGFBP-3 appears the most relevant in humans [17,31]. The effect of IGFBP-3 is to increase the half-life of IGF-1 in the circulation and block its binding to the insulin receptor. In addition, it has been shown to modulate IGF-1 signaling to provide targeted adjustment and fine-tuning under various conditions [29]. As with IGF-1, the bulk of IGFBP-3 is also produced in the liver, although a wide range of other tissues, including reproductive tissues, produce smaller amounts. It is worth noting that hepatocytes are highly specialized cells which do not express IGF or IGFBP receptors thus enabling optimal secretion of these proteins into the circulation. Both IGF-1 and IGFBP-3 are generated under the influence of GH and our earlier study (Study 3) showed significant elevations of both proteins under the influence of adjuvant GH treatment. Whilst there remain many unanswered questions about the wider range of functions for IGFBPs, the modulating influence of IGFBP-3 on IGF-1 is seen to be very important in the interplay of GH and IGF effects within reproductive tissues [17,29]. Hence it has been suggested that the IGF ratio which takes into account both IGF-1 and IGFBP-3 levels, may be a more relevant measure reflecting bioavailability. However, this is not supported from our current study. 
With respect to assessing GH activity, the previous wisdom indicated that, in post-pubertal adults, GH is secreted as a single pulse, during slow-wave sleep in the night/ early morning. Whilst this is more likely in males, pre-menopausal women display more pulses, particularly at the pre-ovulatory phase of their cycle; the frequency and amplitude of these pulses being reflective of their E2 levels. Consistent with this, our recent Study 4 indicates that some women have normal GH levels ( $\geq 3 \mathrm{ng} / \mathrm{mL}$ ) on our tests undertaken between 8am to $10 \mathrm{am}$ in the early follicular phase of the menstrual cycle [21]. Whilst it was the case that the majority of women (73\%) had very low GH levels, the mean was around $1.5 \mathrm{ng} / \mathrm{mL}$ for the $1035 \mathrm{sub}$-fertile women, although the median level was around $0.5 \mathrm{ng} / \mathrm{mL}$, reflecting the high degree of kurtosis in the skewed distribution.

Until now, there has been little published concerning the GH and IGF system among infertile women undertaking ART. Recently 2 studies have been reported from the Cornell Weill Institute in New York, USA. One indicated that IGF-1 levels can predict cancelled cycles during IVF attempts [32] whilst the other indicated IGF-1 could predict OHSS outcomes in poor-responders [33]. The studies implied that high IGF-1 levels had the unfavorable outcomes, but our studies, focusing on pregnancy and live birth outcomes, showed that women with high IGF-1 levels had the most favorable outcomes. Two earlier studies from our PIVET-Curtin collaboration and conducted on women $>40$ years with defined poor-prognosis, demonstrated the up-regulation of GH receptors on oocytes [34] and granulosa cells [35] in association with improved pregnancy outcomes. Mitochondrial activity within oocytes was also improved under the influence of the GH adjuvant which increased and favorably modulated the density of receptors for GH, FSH, LH and bone morphogenetic protein (BMP). Another recent study from Spain independently reports improved oocyte and embryo quality in young women given GH adjuvant for repeated implantation favor causing poor-prognosis 36]. Consistent with these findings, a recent review drawing on both animal and human studies, indicates that the GH/IVF system is very involved in the angiogenesis underlying folliculogenesis in the ovary as well as preparations in the endometrial bed of the uterus favoring embryo implantation [37].

\section{Conclusion}

With the intention of defining those IVF-naïve women who will have a poor prognosis from IVF treatment, we have undertaken a series of studies to examine the relevance of the IGF profile, namely IGF-1, IGFBP-3 and the IGF ratio. This fifth study explores clinical outcomes against a preliminary Assessment Cycle investigation and demonstrates that the best prognosis from a range of clinical parameters including the most important, that of the chance of a live birth, relates to the highest quartile of IGF-1 levels. The IGFBP-3 levels show minimal variation or influence over clinical outcomes. However, the IGF ratio can associate with beneficial clinical outcomes (namely a low IGFBP-3/IGF-1 ratio), but the feature is mostly of borderline significance and never better than the IGF-1 levels alone which demonstrate highly significant associations with oocyte numbers retrieved at OPU and oocyte utilization as well the pregnancy and livebirth outcomes. We believe this new information will enable a more rational approach to exploring the use of adjuvant therapies, particularly that of growth hormone, in the attempt to improve the prognosis for many women who are currently experiencing a poor prognosis within ART programs.

\section{Compliance with ethical standards}

\section{Acknowledgments}

We are grateful for the close working relationship between PIVET $®$ Medical Centre and CLINIPATH $®$ Pathology which carried out the assays involved in the IGF profile and is also accredited by NATA.

\section{Disclosure of conflict of interest}

The entire project has been funded internally at PIVET without any external or commercial contributions. The authors declare no conflict of interest.

\section{Statement of ethical approval}

Reporting of the data was approved under Curtin University Human Ethics Committee approval no. RD_25-10 general approval for retrospective data analysis in 2010, updated in 2015, and again further updated recently, in August 2020.

\section{Statement of informed consent}

PIVET is accredited with both the self-regulatory National Australian Reproductive Technology Committee (RTAC) as well as the Reproductive Technology Council (RTC) of Western Australia. Consent forms received approval under both 
regulatory bodies. The assay laboratory is accredited on an annual basis by the National Australian Testing Authority (NATA).

\section{Author Contributions}

The study was conceived by PIVET Medical Director JLY who established the data base at PIVET Medical Centre with the assistance of IT Consultant and data manager PMH. The first data extractions were undertaken by SZ who was on a sabbatical study period from her facility in Malaysia to which she has now returned. The Data has been further analyses by PMH supported by MDKN who has a managerial role for the Laboratory Assays at PIVET. All authors have assisted with the data analyses as well as the preparation of the Tables and Figures. The manuscript was written by JLY and each of the authors have read and agreed to its content.

\section{References}

[1] Newman JE, Paul RC, Chambers GM 2020. Assisted reproductive technology in Australia and New Zealand. Sydney: National Perinatal Epidemiology and Statistics Unit, the University of New South Wales, Sydney, 2018; 1-83.ISBN-13: 978-0-7334-3941-4

[2] Keane K, Cruzat VF, Wagle S, Chaudhary N, Newsholme P, Yovich J. Specific ranges of anti-Mullerian hormone and antral follicle count correlate to provide a prognostic indicator for IVF outcome. Reprod Biol. 2017; 17: 51-59. Doi: 10.1016/j.repbio.2016.12.002

[3] Mustafa KB, Keane KN, Walz NL, Mitrovic KI, Hinchliffe PM, Yovich JL.Live Birth Rates are satisfactory following multiple IVF treatment cycles in poor prognosis patients. Reprod Biol. 2017; 17: 34-41Doi: 10.1016/j.repbio.2016.11.004

[4] Yovich JL, Alsbjerg B, Conceicao JL, Hinchliffe PM, Keane KN. PIVET rFSH dosing algorithms for individualized controlled ovarian stimulation enables optimized pregnancy productivity rates and avoidance of ovarian hyperstimulation syndrome. Drug Des Devel Ther. 2016; 10: 2561-2573.Doi: 10.2147/DDDT.S104104

[5] Yovich JL. How to Prepare the Egg and Embryo to Maximise IVF Success. In: Monitoring the stimulated IVF cycle. Section II: Stimulation for IVF (Eds: Gabor T Kovacs, Anthony J Rutherford, David K Gardner). Cambridge University Press, Cambridge 2019; 94-120.Doi: 10.1017/9781316756744

[6] Yovich JL, Ye Y, Regan SLP, Keane KN. The evolving concept of poor-prognosis for women undertaking IVF and the notion of growth hormone as an adjuvant; a single-center viewpoint. Front. Endocrinol. 2019; 10: 808:114.Doi: $10.3389 /$ Fendo.2019.00808

[7] Farquhar C. Add-ons for assisted reproductive technology: can we be honest here? Fertil Steril. 2019; 112(6): 971-972.Doi: 10.1016/j.fertnstert.2019.10.010

[8] Annual Capri Workshop Group. IVF, from the past to the future: the inheritance of the Capri Workshop Group. Hum Reprod Open. 2020; 3:1-9.Doi: 10.1093/hropen/hoaa040

[9] Yovich JL, Regan SL, Zaidi SN, Keane KN. The concept of growth hormone deficiency affecting clinical prognosis in IVF. Front Endocrinol. 2019; 10: 650, 1-9. Doi: 10.3389/fendo.2019.00650

[10] Zhang Y, Zhang C, Shu J, Guo J, Chang H-M, Leung PCK, Sheng J-Z, Huang H. Adjuvant treatment strategies in ovarian stimulation for poor responders undergoing IVF: a systematic review and network meta-analysis. Hum Reprod Update. 2020; 26(2): 247-263Doi: 10.1093/humupd/dmz046

[11] Yang P, Wu R, Zhang $\mathrm{H}$. The effect of growth hormone supplementation in poor ovarian responders undergoing IVF or ICSI: a meta-analysis of randomized controlled trials. Reprod Biol Endocrinol. 2020; 18(1):76:1-10.Doi: 10.1186/s12958-020-00632-w.

[12] Stanley T. Diagnosis of growth hormone deficiency in childhood. Curr Opin Endocrinol Diabetes Obes. 2012; 19: 47-52.Doi: 10.1097/MED. 0b013e32834ec952

[13] Gaddas M, Périn L, Le Bouc Y. Evaluation of IGF1/IGFBP3 molar ratio as an effective tool for assessing the safety of growth hormone therapy in small-for-gestational-age, growth hormone-deficient and Prader-Willi children. J Clin Res Pediatr Endocrinol. 2019; 11(3):253-261.Doi: 10.4274/jcrpe.galenos.2019.2018.0277

[14] Allard JB, Duan C. IGF-binding proteins: why do they exist and why are there so many? Front Endocrinol. 2018; 9: 117:1-12.Doi: 10.3389/fendo.2018.00117 
[15] Juul A, Main K, Blum WF, Lindholm J, Ranke MB, Skakkebaek NE. The ratio between serum levels of insulin-like growth factor (IGF)-1 and the IGF binding proteins (IGFBP-1,2, and 3) decreases with age in healthy adults and is increased in acromegalic patients. Clin Endocrinol (Oxf). 1994; 41(1):85-93.Doi: 10.1111/j.13652265.1994.tb03788.x

[16] Molitch ME, Clemmons DR, Malozowski S, Merriam GR, Vance ML. Evaluation and treatment of adult growth hormone deficiency: An Endocrine Society Clinical Practice Guideline. J Clin Endocrinol Metab. 2011; 96(6): 1587-1609.Doi: 10.1210/jc.2011-0179

[17] Olarescu NC, Gunawardene K, Hansen TK, Møller N, Jørgensen JOL. Normal physiology of growth hormone in adults. In: Feingold KR, Anawalt B, Boyce A et al., editors. Endotext [Internet]. South Dartmouth (MA): NCBI Bookshelf; MD Text.com, Inc.; 2000-. 2019; 1-28

[18] Yovich JL, Zaidi S, Nguyen MDK, Hinchliffe PM. Measuring IGF-1 and IGFBP-3 profiles in women seeking assisted reproduction; relationship to clinical parameters (Study 1). J Pers Med. 2020; 10(3): 122, 1-15 Doi: 10.3390/jpm10030122

[19] Yovich JL, Zaidi S, Nguyen MDK, Hinchliffe PM. Measuring IGF-1 and IGFBP-3 profiles in women seeking assisted reproduction; relationship to ovarian reserve parameters (Study 2). GSC Biol \& Pharmaceutical Sciences. 2020; 13(02), 035-053.

[20] Yovich JL, Zaidi S, Nguyen MDK, Hinchliffe PM. Measuring IGF-1 and IGFBP-3 profiles in women seeking assisted reproduction; relationship to serum growth hormone levels (Study 3). GSC Biological and Pharmaceutical Sciences, 2020; 13(03): 032-053.

[21] Yovich JL, Zaidi S, Nguyen MDK, Hinchliffe PM. Measuring IGF-1 and IGFBP-3 profiles in women seeking assisted reproduction; response of women categorised as poor prognosis to growth hormone adjuvant therapy (Study 4). GSC Biological and Pharmaceutical Sciences, 2020; 13(03): 064-078.

[22] Yovich JL, Craft IL. Founding pioneers of IVF: Independent innovative researchers generating livebirths within 4 years of the first birth. Reprod Biol. 2018; 18: 317-323.Doi.org/10.1016/J.repbio.2018.11.004

[23] Yovich JL. Founding pioneers of IVF Update: Independent innovative researchers generating livebirths within 4 years of the first birth. Reprod Biol. 2020; 20: 111-113 Doi.org/10.1016/j.repbio.2019.12.008

[24] Yovich J, Stanger J, Hinchliffe P. Targeted gonadotrophin stimulation using the PIVET algorithm markedly reduces the risk of OHSS. Reprod Biomed Online. 2012; 24(3): 281-292.Doi: 10.1016/j.rbmo.2011.11.014

[25] Kuwayama M, Vajta G, Kato O, Leibo SP. Highly efficient vitrification method for cryopreservation of human oocytes. Reprod Biomed Online. 2005; 11:300-308 Doi: 10.1016/s1472-6483(10)60837-1

[26] Yovich JL, Conceicao JL, Marjanovich N, Ye Y, Hinchliffe PM, Dhaliwal SS, Keane KN. An ICSI rate of $90 \%$ minimizes complete failed fertilization and provides satisfactory implantation rates without elevating fetal abnormalities. Reprod Biol. 2018; 18: 301-311.Doi: 10.1016/j.repbio.2018.05.002

[27] Yovich JL. Stanger JD, Keane KN. Cumulative live birth rate: An outmoded term. JFIV Reprod Med Genet. 2016; 4:165.Doi:10.4172/2375-4508.1000165

[28] Hammer $\emptyset$, Harper DAT, Ryan PD. PAST: Paleontological Statistics software package for education and data analysis. Palaentologia Electronica 2001; 4(1): 1-9.

[29] Ipsa E, Cruzat VF, Kagize JN, Yovich JL, Keane KN. Growth Hormone and Insulin-like growth factor in reproductive tissues. Front Endocrinol. 2019; 777: 1-14.Doi: 10.3389/fendo.2019.00777

[30] Hull KL, Harvey S. Growth hormone and reproduction: a review of endocrine and autocrine, paracrine interactions. Int J Endocrinol. 2014; Article ID 234014: 1-24.Doi: 10.1155/2014/234014

[31] Steyn FJ, Tolle V, Chen C, Epelbaum J. Neuroendocrine regulation of growth hormone secretion. Compr Physiol. 2016; 6(2): 687-735.Doi: 10.1002/cphy.c150002. PMID: 27065166.

[32] Nasioudis D, Minis E, Irani M, Kreines F, Witkin SS, Spandorfer SD. Insulin-like growth factor-1 and soluble FMSlike tyrosine kinase-1 prospectively predict cancelled IVF cycles. J Assist Reprod Genet. 2019; 36(12): $2485-2491$. Doi: 10.1007/s10815-019-01618-3.

[33] Man L, Lekovich J, Canon C, Rosenwaks Z, James D. Cycle day-2 insulin-like growth factor-1 serum levels as a prognostic tool to predict controlled ovarian hyperstimulation outcomes in poor responders. Fertil Steril. 2020; 113: 1205-1214. Doi.org/10.1016/j.fertnstert.2020.02.111 
[34] Weall BM, Al-Samerria S, Conceicao J, Yovich JL, Almahbobi G. A direct action for GH in improvement of oocyte quality in poor-responder patients. Reproduction 2015; 149:147-154.Doi: 10.1530/rep-14-0494

[35] Regan SLP, Knight PG, Yovich JL, Arfuso F, Dharmarajan A. Growth hormone during in vitro fertilization in older women modulates the density of receptors in granulosa cells, with improved pregnancy outcomes. Fertil Steril. 2018; 110: 1298-309.Doi: 10.1016/j.fertnstert.2018.08.018

[36] Tesarik J, Galán-Lázaro M, Conde-López C, Chiara-Rapisarda AM, Mendoza-Tesarik R. The effect of GH administration on oocyte and zygote quality in young women with repeated implantation failure after IVF. Front Endocrinol. 2020; 11:519572:1-7 Doi: 10.3389/fendo.2020.519572

[37] Devesa J, Caicedo D. The role of growth hormone on ovarian functioning and ovarian angiogenesis. Front Endocrinol. 2019; 10:1-16.Doi: 10.3389/fendo.2019.00450. 